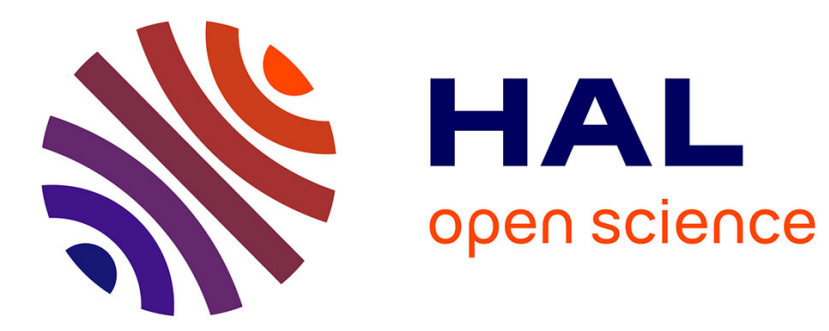

\title{
Shear-thinning mediation of elasto-inertial Taylor-Couette flow
}

Tom Lacassagne, Neil Cagney, Stavroula Balabani

\section{To cite this version:}

Tom Lacassagne, Neil Cagney, Stavroula Balabani. Shear-thinning mediation of elasto-inertial TaylorCouette flow. Journal of Fluid Mechanics, 2021, 915, 10.1017/jfm.2021.104 hal-03185794

\section{HAL Id: hal-03185794 \\ https://hal.science/hal-03185794}

Submitted on 19 Nov 2021

HAL is a multi-disciplinary open access archive for the deposit and dissemination of scientific research documents, whether they are published or not. The documents may come from teaching and research institutions in France or abroad, or from public or private research centers.
L'archive ouverte pluridisciplinaire HAL, est destinée au dépôt et à la diffusion de documents scientifiques de niveau recherche, publiés ou non, émanant des établissements d'enseignement et de recherche français ou étrangers, des laboratoires publics ou privés. 


\title{
Shear-thinning mediation of elasto-inertial Taylor-Couette flow
}

\author{
Tom Lacassagne ${ }^{1,3} \dagger$, Neil Cagney ${ }^{2}$, and Stavroula Balabani ${ }^{1} \ddagger$ \\ ${ }^{1}$ Flume, Department of Mechanical Engineering, University College London (UCL), London, \\ WC1E 7JE, UK \\ ${ }^{2}$ School of Engineering and Materials Science, Queen Mary University of London, UK \\ ${ }^{3}$ IMT Lille Douai, CERI Energie Environnement, Université de Lille, F-59000, Lille, France \\ (Received xx; revised xx; accepted xx)
}

We study the shear-thinning mediation of elasto-inertial transitions in Taylor-Couette flow of viscoelastic polymer solutions. Two types of high molecular weight polymers are used at various concentrations and in water-glycerol solvents of various viscosities. This allows us to access a wide range of elastic numbers and effective shear-thinning indices. Conservative ramp up (slow acceleration of the inner cylinder and subsequent increase in Reynolds number) and steady state (constant rotation speed) experiments are performed, in which the flow is monitored continuously using flow visualisation. Depending on the shear-thinning and elastic properties of the working fluid, very different behaviours are observed. In almost constant-viscosity fluids (Boger fluids), or shear-thinning fluids with significant elasticity, the flow transitions from purely azimuthal Couette flow (CF) to a highly chaotic flow state referred to as elasto-inertial turbulence (EIT) via Taylor Vortex Flow (TVF) and elasto-inertial waves (RSW). When the degree of shear-thinning is increased and elasticity reduced, elastic waves or EIT may fade to a Wavy Taylor Vortex Flow (WTVF) with increasing inertia. Significant shear-thinning leads to a delay in the onset of EIT. Remarkably, in some highly shear-thinning cases, even with a significant elasticity, elastic flow features (EIT, RSW) are completely suppressed, and the flow exhibit a "Newtonian-like" transition sequence (CF-TVF-WTVF). Shear-thinning acts to modify, delay, or even completely suppress elasto-inertial behaviours (RSW, EIT), that would otherwise have existed in the absence of shear-thinning. It is, thus, possible to induce various hydrodynamic regimes by tuning the relative degrees of shear-thinning, elasticity, and inertia.

Key words: Viscoelasticity, Shear-thinning, Taylor-Couette

\section{Introduction and background}

Taylor-Couette flow (TCF) occurs in the gap between two concentric cylinders with one or both rotating. It has been of sustained interest to the fluid mechanics community, rheologists, process engineers and mathematicians over the past century (Taylor 1923). Despite its simple geometrical configuration, Taylor-Couette flow of Newtonian fluids displays a vast array of complex dynamics. This includes a wide variety of steady, unsteady, chaotic or turbulent flow states (Andereck et al. 1986; Coles 1965; Dutcher \&

$\dagger$ Email address for correspondence: tom.lacassagne@imt-lille-douai.fr

$\ddagger$ Email address for correspondence: s.balabani@ucl.ac.uk 
Muller 2009; Akonur \& Lueptow 2003; Grossmann et al. 2016; Gul et al. 2018), occurring when either one or two cylinders rotate, in contra- or co-rotation. In the most common case in which only the inner cylinder rotates and the outer cylinder is fixed, the flow can be characterised using a single Reynolds number:

$$
\operatorname{Re}=\rho \Omega r_{i} d / \mu
$$

where $\rho$ is the fluid density, $\mu$ the dynamic viscosity, $\Omega$ the inner cylinder rotation speed and $\mathrm{d}=r_{o}-r_{i}$ the gap width, i.e. the difference between the inner and outer cylinder radii, $r_{i}$ and $r_{o}$ respectively. The system geometry can be characterised using two nondimensional parameters: the radius ratio $\eta=r_{i} / r_{o}$ and the aspect ratio $\mathrm{AR}=L / d$ where $\mathrm{L}$ is the cylinder length. The first parameter describes the gap width and streamline curvature in a simple shear flow, both having an influence on the stability threshold for Newtonian (Taylor 1923) but also non-Newtonian fluids (Larson et al. 1990; Pakdel \& McKinley 1996).

At low Re in Newtonian fluids, a purely azimuthal and uniform shear flow exists in the gap, known as the circular Couette flow $(\mathrm{CF})$. This flow becomes unstable once a critical Re is reached, and a series of toroidal counter-rotating vortices form in the annulus: this is known as the Taylor vortex flow (TVF). The number of counter rotating vortices along the gap corresponds to the spatial wavelength of the instability, $\lambda$. A further increase in Re causes this state to become unstable and undergo sinusoidal axial oscillations (Wavy Tayor Vortex Flow, WTVF), making it non-axisymmetric. Additional frequencies appear as Re is further increased, leading to a more complex wavy flow (Coughlin \& Marcus $1992 a, b)$ which ultimately becomes turbulent (Grossmann et al. 2016).

Because of this rich set of dynamic states in simple fluids, Taylor-Couette flow has been widely used as a means to study flow transitions and instabilities in more complex systems, such as non-Newtonian fluids (Fardin et al. 2014), frequently encountered in industrial applications (Chhabra \& Richardson 1999). Polymer solutions (Larson \& Desai 2015) or Wormilke Micellar Solutions (WMS) (Divoux et al. 2016) are useful model fluids to describe the behaviour of such systems. The latter have received increased attention in recent years (Fardin et al. 2010, 2012; Perge et al. 2014; Mohammadigoushki \& Muller 2017; Hopkins et al. 2020; Haward et al. 2020) while the former have been extensively used in the literature for several decades (see remainder of section 1), and will also be employed in this study. Two of their most common non-Newtonian properties are shearthinning and viscoelasticity. The former leads to a dynamic viscosity $\mu$ that depends on the shear rate $\dot{\gamma}$ (e.g. in a power law fashion, $\mu \sim \dot{\gamma}^{n-1}$ with $n$ the flow index). Elasticity confers a "solid-like" behaviour to the fluid, with a complex shear modulus $G *$ that comprises an elastic part $G^{\prime}$ and a viscous part $G^{\prime \prime}$ such as $G^{*}=G^{\prime}+i G^{\prime \prime}$. This property is characterized by the existence of an elastic/relaxation time-scale of the fluid, $t_{e}$. Such properties are influenced by many factors including the molecular weight, polymer concentration, conformation of the polymer chains, and entanglement (Larson \& Desai 2015). By using low concentrations of high molecular weight linear polymers in a sufficiently viscous solvent, one may produce "Boger" fluids (Boger 1977; James 2009), i.e. elastic fluids for which the polymer contribution to the viscosity is small compared to the solvent viscosity, and for which the apparent viscosity is thus constant (Newtonian). Equally, by selecting different polymers and solvent viscosities, one can vary elasticity and shear-thinning almost independently, exploring the relative influence of these parameters on TCF over a broad parameter space. 


\subsection{Elastic and elasto-inertial Taylor-Couette flows}

The degree to which a fluid has an elastic response to the flow is expressed by the Weissenberg number, Wi. In a Taylor-Couette system, this number can be defined as $\mathrm{Wi}=t_{e} / \dot{\gamma}$, where $\dot{\gamma}=\Omega r_{i} / d$ is the nominal shear-rate in the gap. The competition between elastic and viscous effects is then expressed by the elastic number:

$$
\mathrm{El}=\frac{\mathrm{Wi}}{\mathrm{Re}}=\frac{t_{e}}{t_{v}}=\frac{t_{e} \mu}{\rho d^{2}},
$$

where $t_{v}=\rho d^{2} / \mu$ is the viscous time-scale. El only depends on the fluid and geometric properties. Based on its value, the fluid can be classified as weakly elastic $\left(\mathrm{El}<10^{-2}\right)$, moderately elastic $\left(10^{-2}<\mathrm{El}<1\right)$, or highly elastic $(\mathrm{El}>1)$ (Latrache et al. 2016; Dutcher \& Muller 2011, 2013). In the range of low elasticity (i.e., El $\ll 1$ ) and with increasing Re, transitions similar to Newtonian fluids have been recovered (Crumeyrolle et al. 2002; Dutcher \& Muller 2011).

At vanishing Re but moderate or high El, a purely elastic CF-TVF transition exists (Larson et al. 1990), and flows may even exhibit a chaotic behaviour (Groisman \& Steinberg 2004; Fardin et al. 2010, 2012; Mohammadigoushki \& Muller 2017), which is called elastic "turbulence", despite the relative absence of inertia (Larson 2000), as it shows chaotic and disordered features usually attributed to turbulence (Groisman \& Steinberg 2000; Varshney \& Steinberg 2019; Steinberg 2019).

At both non-negligible Re and El values, elasto-inertial instabilities (primary or secondary) occur (Steinberg \& Groisman 1998). They involve non-axisymmetric flow states and standing or travelling elastic waves such as ribbons (RIB) (Baumert \& Muller 1999; Latrache et al. 2016), spiral vortex flow (SVF) (Avgousti \& Beris 1993; Mohammadigoushki \& Muller 2017) and rotating spiral waves (RSW) (Groisman \& Steinberg 1996; Thomas et al. 2006; Dutcher \& Muller 2013; Lacassagne et al. 2020). Upon increasing Re or El, such pre-chaotic states are replaced by unsteady, chaotic flow states, that have been called disordered oscillations (DO) (Groisman \& Steinberg 1996), defect mediated turbulence (DMT) or spatio-temporal intermittency (STI) (Latrache et al. $2012,2016)$. They all consist in a gradual transition to elasto-inertial turbulence (EIT) (Dutcher \& Muller 2013; Liu \& Khomami 2013; Perge et al. 2014; Lacassagne et al. 2020). Elasto-inertial (and elastic) turbulence induces spatial and temporal chaos in fluids that would otherwise have been flowing in laminar states without elasticity, promoting mixing and momentum transfer (Groisman \& Steinberg 2004).

\subsection{Shear-thinning Taylor-Couette flows with negligible elasticity}

In shear-thinning fluids with negligible elasticity, the typical transition pattern is "Newtonian-like": CF-TVF-WTVF (Escudier et al. 1995; Bahrani et al. 2015; Topayev et al. 2019; Cagney \& Balabani 2019b). In the small gap limit, a decrease in the critical Reynolds number for the formation of Taylor vortices $\operatorname{Re}_{c}^{T V F}$ (destabilisation) occurs with increased shear-thinning. This has been observed both experimentally (Cagney et al. 2020; Cagney \& Balabani 2019a; Escudier et al. 1995) and numerically (Ashrafi \& Khayat 2000; Caton 2006). In the large gap limit, shear-thinning has a non-monotonic effect on $\operatorname{Re}_{c}^{T V F}$, (Sinevic et al. 1986; Lockett et al. 1992; Cagney \& Balabani 2019a; Alibenyahia et al. 2012).

The spatial wavelength of the TVF has been found to increase with increased shearthinning, as observed in experiments and the linear stability analysis by Bahrani et al. (2015) (in a large gap flow cell), laser-Doppler velocimetry measurements by Escudier et al. (1995), or particle image velocimetry (PIV) in Cagney \& Balabani (2019b). From 
the two later works and recent numerical simulations by Topayev et al. (2019) and Alibenyahia et al. (2012), shear-thinning was found to modify the structure of the Taylor vortices, by making individual vortices asymmetric, and increasing the amplitudes of the travelling waves at the inward and outward jets. Sparse data on the critical Re value for the TVF to WTVF (Bahrani et al. 2015; Cagney et al. 2020) suggest that the TVF to WTVF transition happens at lower Re values with increased shear-thinning. Shearthinning also alters the structure of the wavy vortices (Cagney \& Balabani 2019b; Cagney et al. 2020).

\subsection{Combined shear-thinning and elasticity}

The effects of shear-thinning and elasticity have been studied separately in the literature, with elastic fluids having received more attention to date. There is still a lack of experimental work on fluids combining the two properties. In studies focused on elastic fluids, shear-thinning, when reported, is generally mild and typically neglected. However, even moderate shear-thinning can have a significant impact on the base flow structure, and thus on the development of elastic instabilities. The onset of the primary instability is found at lower critical Re values than in Newtonian fluids, regardless of shear-thinning (Larson et al. 1994; Crumeyrolle et al. 2002; Dutcher \& Muller 2011, 2013; Schaefer et al. 2018; Cagney \& Balabani 2019b; Cagney et al. 2020). The main challenge resides in describing higher order, higher Re flow transitions.

Latrache and co-workers performed a series of experimental works using shear-thinning and viscoelastic PolyEthylene Oxide (PEO) solutions in low viscosity solvents. They reported several types of transition patterns, from Newtonian-like (Crumeyrolle et al. 2002 ) at $\mathrm{El}<0.03$, to "elastic-like" with RIB (Crumeyrolle et al. 2002, 2005) and EIT states (Latrache et al. 2012, 2016; Abcha et al. 2018). In Crumeyrolle et al. (2005) and Latrache et al. (2016), defect mediated turbulence was reported, entailing a local collision of RIB waves leading to a spatio-temporal heterogeneity of the RIB map. The multiplication of such features ultimately leads to a fully disordered flow that can be labelled DO or EIT. In a recent work (Lacassagne et al. 2020), we reported an analog behaviour of elasticity promoting local anomalies in the flow, this time in a non-shearthinning Boger fluid. The mechanism was yet different from DMT, as it consisted in merging and splitting of base Taylor vortices crossed by axial RSW waves, instead of defects in RIB amplitude. It thus appears that the mechanisms for transition to EIT are greatly affected by the shear-thinning behaviour of the fluid.

Dutcher \& Muller (2009, 2011, 2013) also used shear-thinning and elastic PEO solutions, to study the effects of weak and intermediate elasticity. Comparing their results with those of Crumeyrolle et al. (2002), the authors argued that the difference in the ratio of polymer and solvent viscosity may explain the different flow transitions observed at similar El values. This polymer-to-solvent viscosity ratio is intrinsically related to the shear-thinning property: the higher this ratio, the more the polymer contributes to the overall viscosity and the stronger the shear-thinning level is likely to become.

In our recent work (Cagney et al. 2020), xanthan gum (XG) solutions with systematically increasing concentration were produced, yielding fluids with both increasing shearthinning and elastic properties. Even at high El values, the Newtonian-like transition pattern associated with shear-thinning fluids was retrieved, and no typical features of elastic behaviours (RSW, RIB, EIT) were reported. In this study, as in those of Latrache and co-workers, disentangling the effects of shear-thinning and elasticity was difficult, as both properties were varied simultaneously with the polymer concentration. Moreover, many previous studies examining TC flow of XG solutions did not fully characterise the rheology and assumed negligible elasticity, making it challenging to distinguish the effects 
of either phenomenon on the flow (Elçiçek \& Güzel 2020a,b; Bahrani et al. 2015; Masuda et al. 2017).

Finally, in TC flows of shear-thinning and elastic WMS, travelling vortices (SVF), standing waves (RIB) and transition to EIT have been reported. In Perge et al. (2014) the typical transition pattern is CF-RIB-EIT in a significantly shear-thinning fluid $(n=0.45)$ at $\mathrm{El} \sim 1$. In Mohammadigoushki \& Muller (2017), travelling vortices that could be SVF were reported, as well as a transition to EIT.

Experimental data on Taylor-Couette flow of fluids that are both shear-thinning and viscoelastic is inconclusive regarding how shear-thinning affects elastic instabilities and transitions. It appears that elastic fluids may exhibit a Newtonian-like transition pattern, in the low elasticity limit (Dutcher \& Muller 2011; Crumeyrolle et al. 2002), but also at high El when they are also shear-thinning (Cagney et al. 2020), suggesting that shear-thinning may act to reduce or suppress elastic instabilities. Such a shear-thinning modification of elastic effects has also been observed in other types of flows (PEO in serpentine micro-channel, Casanellas et al. (2016), WMS flow past micro-pins, Haward et al. (2020)). On the other hand, shear-thinning is known to be conditional for some elastic instabilities to develop, for example in viscoelastic (pressure driven) channel flows (Bodiguel et al. 2015; Barlow et al. 2019).

In this work, we thus aim at disentangling the effects of shear-thinning and elasticity on TCF transitions and patterns in polymer solutions. We present flow visualisation measurements of TCF for polymer solutions with variable degrees of elasticity and shearthinning. The remainder of the paper is structured as follows. Firstly, the experimental system is described in section 2 . The results of flow visualisation experiments are then reported in section 3 . In section 4 , the combined effects of shear-thinning and elasticity on transitions to TVF and WTVF and EIT, including potential suppression of the latter, are discussed, and some concluding remarks can be found in section 5 .

\section{Experiments}

\subsection{Taylor-Couette cell}

The experiments were performed in a custom made Taylor-Couette flow cell. It comprised an outer cylinder made of an acrylic pipe, mounted vertically between two acrylic plates, and a black PTFE inner cylinder. The latter had a conical tip complementary to a dent in a bottom plate which allowed to reduce bottom friction while, together with a ball bearing at the top lid, ensuring alignment between inner and outer cylinder. The top and bottom lids were stationary, with $\mathrm{a} \sim 3 \mathrm{~mm}$ gap between lid and cylinder at both ends. The cylinder was driven from the top using a stepper motor (SmartDrive Ltd, Cambridge, U.K.), the rotation of which could be controlled to a high degree of precision by a 52000 microstep/revolution controller (SmartDrive Ltd, Cambridge, U.K.). The inner chamber, which was completely filled with working fluid, had an axial length of $L=135 \mathrm{~mm}$. The inner and outer radii of the flow cell were $21.66 \mathrm{~mm}$ and $27.92 \mathrm{~mm}$, respectively. The radius ratio was thus $\eta=0.77$, the gap width was $\mathrm{d}=6.26 \mathrm{~mm}$, and the aspect ratio was $\mathrm{AR}=21.56$. The geometric parameters are sketched on figure 1 .

The flow section was enclosed in a rectangular chamber in which water was recirculated via a temperature bath to ensure that the temperature remained constant and close to $20^{\circ} \mathrm{C}$ throughout experiments. The temperature within the flow cell was measured immediately before and after each experiment, and it was found to vary by less than $0.2^{\circ} \mathrm{C}$. 


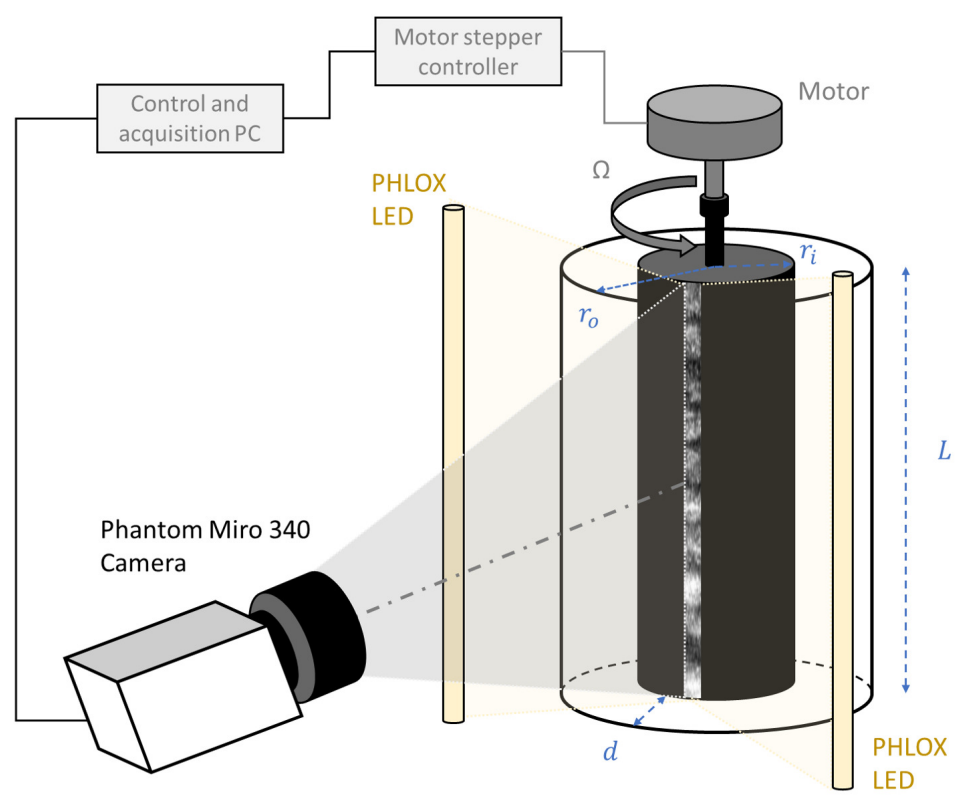

Figure 1: Sketch of the test-section and flow visualisation system.

\subsection{Polymer solutions and rheological characterisation}

Optically clear shear-thinning and viscoelastic polymer solutions were prepared by dissolving XG (Sigma Aldrich) into a mixture of glycerol and deionized water. The weightaveraged molecular mass $M_{w}$ was measured by size exclusion chromatography and found equal to $1.76 \times 10^{6}$ g.mol ${ }^{-1}$ (Cagney et al. 2020).

Various degrees of elasticity and shear-thinning of the XG solutions were achieved by varying solution composition (polymer concentration and glycerol volume fractions). For comparison, a Boger fluid was prepared by dissolving small concentrations of PolyAcrylamide (PAAM, $M_{w}=5.5 \times 10^{6} \mathrm{~g} . \mathrm{mol}^{-1}$, Sigma Aldrich) into deionized water-glycerol mixtures. Polymers were progressively added to the solvent, left to dissolve overnight without any agitation, to prevent polymer chain destruction in the preparation process, and the mixture was finally homogenised by gentle shaking. Polymer type, concentration, and solvent composition and viscosity for each working fluid used in this work are listed in table 1. The sample naming convention used throughout the paper is the following: letters correspond to the polymer used (XG for xanthan gum, $\mathrm{P}$ for polyacrylamide, $\mathrm{N}$ for none), the last two digits after the "-" sign to the glycerol volume fraction (e.g. -72 for $72 \%$ ) and the middle number, if present, to the polymer concentration in ppm (parts per million in weight).

The steady-shear rheology of all working fluids was measured using a rotational rheometer (ARES, TA Instruments) equipped with a Couette geometry (inner radius $32 \mathrm{~mm}$, outer radius $34 \mathrm{~mm}$ ) in steady shear mode. All XG samples exhibit shearthinning. The shear viscosity of PAAM sample remains almost constant for a wide range of shear rates. Flow curves (viscosity versus shear rate) for all working fluids are shown in figure 2. The shear-thinning data can be described using a Carreau model

$$
\mu(\dot{\gamma})=\mu_{\infty}+\left(\mu_{0}-\mu_{\infty}\right)\left(1+\left(t_{c} \dot{\gamma}\right)^{2}\right)^{\left(n_{c}-1\right) / 2}
$$




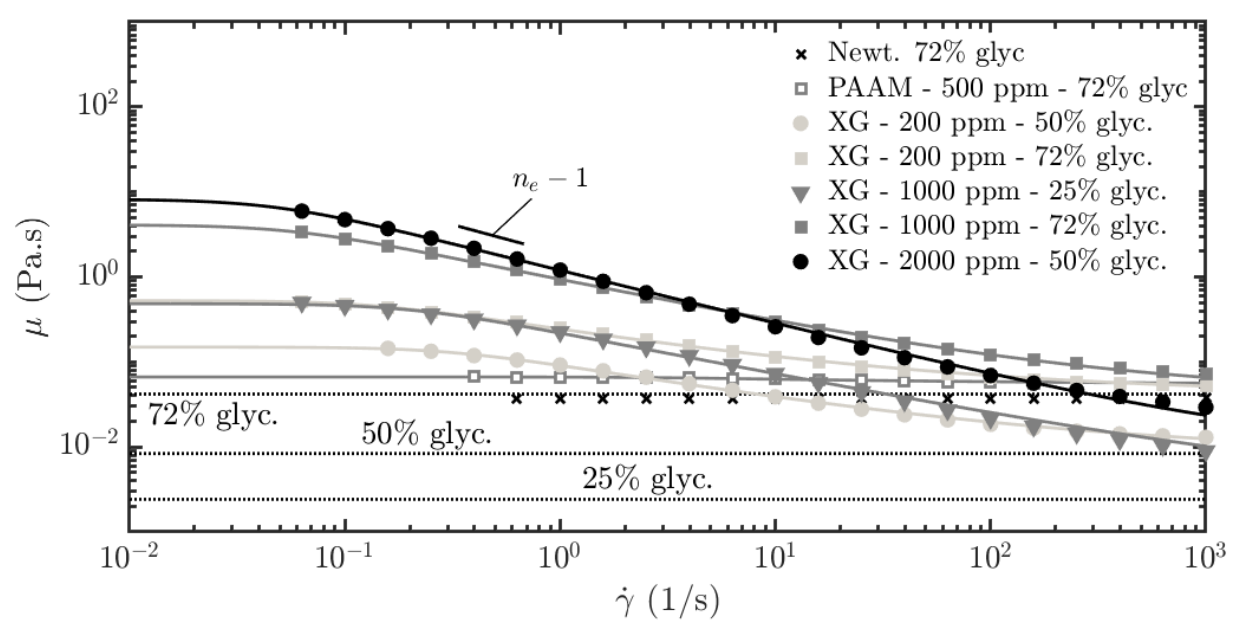

Figure 2: Viscosity as a function of shear rate for polymer solutions (filled symbols for $\mathrm{XG}$, open symbols for PAAM) at various concentrations and in solvents of three different glycerol volume fractions, $25 \%$ (triangles), $50 \%$ (circles) and $72 \%$ (squares). Expected solvent constant viscosities $\mu_{s}$ are derived from Volk \& Kähler (2018) and plotted as dotted lines. The measured viscosity for the reference Newtonian case $(72 \%$ glycerol in water) is represented by black crosses.

\begin{tabular}{|c|c|c|c|c|c|c|c|c|c|c|c|}
\hline ID & Pol. & $\begin{array}{l}\mathrm{C} \\
\mathrm{ppm}\end{array}$ & $\begin{array}{l}\text { Gly. } \\
\% v\end{array}$ & $\begin{array}{l}\rho \\
\mathrm{Kg} \cdot \mathrm{m}^{-3}\end{array}$ & $\begin{array}{l}\mu_{s} \\
\text { Pa.s }\end{array}$ & $\begin{array}{l}\mu_{0} \\
\text { Pa.s }\end{array}$ & $\begin{array}{l}\mu_{\infty} \\
\text { Pa.s }\end{array}$ & $n_{c}$ & $\begin{array}{l}t_{c} \\
\mathrm{~s}\end{array}$ & $\frac{\mu_{0}}{\mu_{\infty}}$ & $\begin{array}{l}t_{e} \\
\mathrm{~s}\end{array}$ \\
\hline $\mathrm{N}-72$ & - & 0 & 0.72 & 1198 & 0.0363 & - & - & - & - & 1.0 & - \\
\hline P500-72 & PAAM & 500 & 0.72 & 1198 & 0.0363 & 0.0573 & 0.0425 & 0.6622 & 0.2268 & 1.35 & 0.1939 \\
\hline XG200-72 & $\mathrm{XG}$ & 200 & 0.72 & 1198 & 0.0363 & 0.5027 & 0.0419 & 0.5734 & 6.949 & 12.0 & 0.1257 \\
\hline XG200-50 & $\mathrm{XG}$ & 200 & 0.50 & 1142 & 0.0083 & 0.1291 & 0.0084 & 0.586 & 2.895 & 15.3 & 0.2194 \\
\hline XG1000-72 & $\mathrm{XG}$ & 1000 & 0.72 & 1198 & 0.0363 & 3.674 & 0.0419 & 0.5009 & 16.48 & 87.7 & 11.46 \\
\hline XG1000-25 & $\mathrm{XG}$ & 1000 & 0.25 & 1072 & 0.0024 & 0.5199 & 0.0024 & 0.5386 & 6.9133 & 214 & 2.536 \\
\hline XG2000-50 & $\mathrm{XG}$ & 2000 & 0.50 & 1142 & 0.0083 & 8.090 & 0.0084 & 0.3709 & 22.20 & 967 & 50.24 \\
\hline
\end{tabular}

Table 1: Polymer solutions and their rheological properties at $20^{\circ} \mathrm{C}$. The sample labelling scheme is the following (ID column): XXiii-jj where XX is the polymer molecule used ( $\mathrm{N}$ if no polymer), iii is the polymer concentration in ppm (empty if no polymer), and jj is the glycerol volume fraction in the solvent in volume $\%$.

where $\mu_{\infty}$ and $\mu_{0}$ are viscosity plateau values at infinite and zero shear respectively, $t_{c}$ is the characteristic Carreau time scale, and $n_{c}$ is the Carreau flow index. The fitted expressions are plotted on figure 2 as full lines. The estimated parameters for each fluid are also displayed in table 1 . The ratio $\mu_{0} / \mu_{\infty}$ (reported in the last column of table 1 ) indicates the difference between so called Boger fluids, for which the viscosity can be assumed constant with $\mu_{0} / \mu_{\infty} \sim 1$, and shear-thinning fluids for which $\mu_{0} / \mu_{\infty} \gg 1$. For a given fluid, the degree of shear-thinning (slope of the curve) was shear-rate dependent. This means that the effective shear-thinning behaviour varied as the rotation speed was varied. It is thus convenient to define a shear rate dependent, "effective" flow index from the local slope of the flow curve in a log-log space (sketched on figure 2, see CoronadoMatutti et al. (2004); Cagney \& Balabani (2019b); Cagney et al. (2020)): 


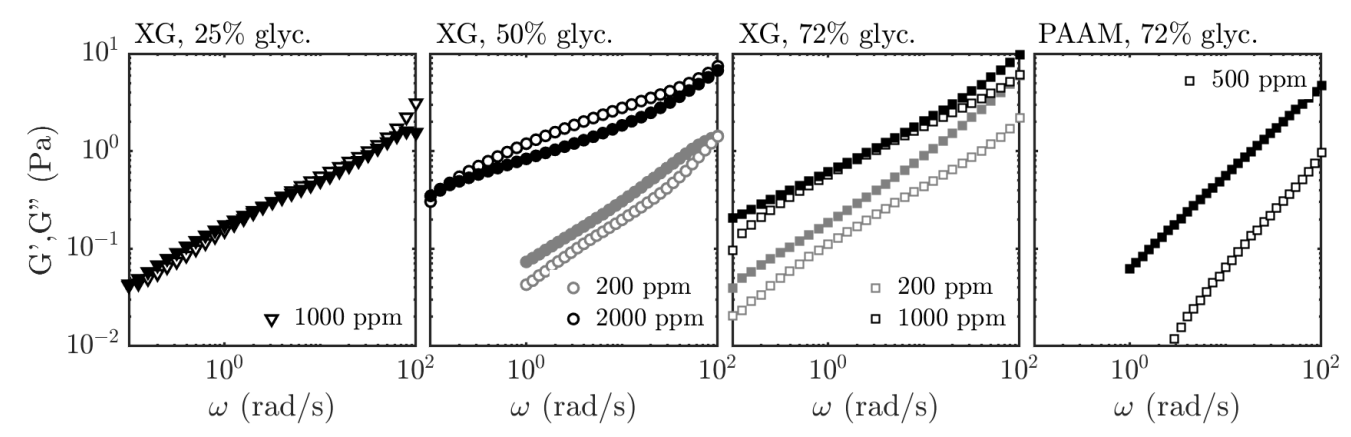

Figure 3: G' (open symbols) and G" (filled symbols) moduli for XG and PAAM solutions at various concentrations and in various glycerol water solvents.

$$
n_{e}=\frac{\partial \log (\mu)}{\partial \log (\dot{\gamma})}+1,
$$

where $n_{e}$ is equivalent to the previously defined flow index $n$, but accounts for the nonpower-law behaviour via shear-rate dependency. An effective flow index close to unity means that the fluid behaves as a Newtonian fluid, and the effective flow index decreases with increasing shear-thinning behaviour. While $n_{c}$ may vary non-monotonically with polymer concentration, $n_{e}$ always decreases with increasing polymer concentration at a given shear-rate and for a given solvent, and is a better indication of the degree of shear-thinning in each fluid (Cagney et al. 2020).

The elastic properties of the samples were quantified by oscillatory shear experiments on the same rheometer and geometry. The evolution of $G^{\prime}$ and $G^{\prime \prime}$ with oscillation frequency were measured for $\gamma=5 \% . G^{\prime}$ and $G$ " frequency sweeps are shown in figure 3 .

For this study, a common way to estimate elastic time-scales for the various types of fluids used and compare them, regardless of the solvent contribution to viscosity, was needed. To do so, the loss modulus curves from figure 3 were corrected by removing the contribution of the solvent viscosity (Zirnsak et al. 1999), such that

$$
\tilde{G^{\prime \prime}}=G^{\prime \prime}-\mu_{s} \omega .
$$

The elastic time scale could then be obtained from the crossover frequency $\tilde{\omega}_{c}$ between G' and $\tilde{G}$ " curves (not shown in figure 3 for the sake of readability), such that $t_{e}=2 \pi / \tilde{\omega}_{c}$. Doing so, one could access the polymer time-scale regardless of the solvent used (even in fluids for which the solvent contribution to the overall viscosity is large compared to the polymer one, and for which no crossover between G' and G" can be observed in figure 3). Estimated time scales agreed with those estimated from Zimm approximation or multi-mode Maxwell fitting on G' and G" curves (Öztekin et al. 1994).

\subsection{Flow visualisation experiments}

The flow was visualized by adding reflective mica flakes to the fluid (Cornelissen \& Son, Pearl Lustre Pigments), at a volume fraction of the order of $10^{-4}$, similar to previous studies (Cagney \& Balabani 2019b; Cagney et al. 2020). Images of a vertical strip along the cylinder's axial dimension were acquired by a Phantom Miro 340 camera (see figure 1 ). The frame rate $f_{s}$ was adjusted throughout the experiments in order to achieve high temporal resolution of the Reynolds number range studied. For ramp up and steady 
Sample Test $f_{s} \quad 1 / \Delta R e \quad \Omega_{\max } \bar{n}_{e} \quad \overline{\mathrm{El}} \quad \mathrm{d} \Omega / \mathrm{d} t \quad \Gamma_{0} \quad$ Transition sequence

\begin{tabular}{|c|c|c|c|c|c|c|c|c|c|}
\hline & & $\mathrm{Hz}$ & & $\mathrm{s}^{-1}$ & & & $\mathrm{~s}^{-2}$ & & \\
\hline N72 & 1 & 75 & 224.32 & 66.96 & 1.0 & 0 & 0.07463 & 0.4338 & \multirow{2}{*}{ CF-TVF-WTVF } \\
\hline N72 & 2 & 30 & 201.88 & 66.96 & 1.0 & 0 & 0.03317 & 0.1928 & \\
\hline P500-72 & 1 & 200 & 225.56 & 99.23 & 0.96 & 0.19 & 0.2911 & 0.7705 & \multirow{5}{*}{$\begin{array}{l}\text { CF-TVF-RSW- } \\
\text { EIT }\end{array}$} \\
\hline P500-72 & 2 & 200 & 225.56 & 99.23 & 0.96 & 0.19 & 0.2911 & 0.7705 & \\
\hline P500-72 & 3 & 90 & 253.77 & 99.23 & 0.96 & 0.19 & 0.1164 & 0.3082 & \\
\hline P500-72 & 4 & 90 & 255.43 & 87.64 & 0.97 & 0.21 & 0.1023 & 0.3473 & \\
\hline P500-72 & 5 & 90 & 255.43 & 87.64 & 0.97 & 0.21 & 0.1023 & 0.3473 & \\
\hline XG200-72 & 1 & 62 & 236.4 & 108.0 & 0.85 & 0.88 & 0.09161 & 0.2047 & \multirow{2}{*}{$\begin{array}{l}\text { CF-TVF-(RSW)- } \\
\text { EIT-WTVF }\end{array}$} \\
\hline XG200-72 & 2 & 90 & 258.4 & 107.1 & 0.85 & 0.17 & 0.1208 & 0.2743 & \\
\hline XG200-50 & 1 & 42 & 178.9 & 36.80 & 0.75 & 0.080 & 0.02770 & 0.5330 & \multirow{3}{*}{$\begin{array}{l}\text { CF-TVF-RSW- } \\
\text { WTVF }\end{array}$} \\
\hline XG200-50 & 2 & 42 & 178.9 & 36.80 & 0.75 & 0.080 & 0.02770 & 0.5330 & \\
\hline XG200-50 & 3 & 62 & 281.1 & 36.18 & 0.73 & 0.14 & 0.02549 & 0.5078 & \\
\hline XG1000-72 & 1 & 250 & 157.5 & 147.5 & 0.72 & 35 & 0.7448 & 0.8924 & \multirow{4}{*}{ CF-EIT } \\
\hline XG1000-72 & 2 & 250 & 150.1 & 153.3 & 0.71 & 13 & 0.8125 & 0.9013 & \\
\hline XG1000-72 & 3 & 50 & 157.5 & 147.5 & 0.72 & 35 & 0.14895 & 0.1785 & \\
\hline XG1000-72 & 4 & 90 & 270.1 & 153.3 & 0.71 & 13 & 0.1625 & 0.1803 & \\
\hline XG1000-25 & 1 & 30 & 199.9 & 46.84 & 0.58 & 1.2 & 0.02229 & 0.2648 & CF-TVF-WTVF \\
\hline XG2000-50 & 1 & 94 & 162.2 & 76.50 & 0.46 & 67 & 0.1385 & 0.6168 & \multirow{4}{*}{$\begin{array}{l}\text { CF-TVF-SVF- } \\
\text { RSW-EIT }\end{array}$} \\
\hline XG2000-50 & 2 & 42 & 212.5 & 95.02 & 0.56 & 74 & 0.05875 & 0.1696 & \\
\hline XG2000-50 & 3 & 200 & 235.5 & 82.75 & 0.46 & 67 & 0.2198 & 0.8366 & \\
\hline XG2000-50 & 4 & 200 & 235.5 & 82.75 & 0.46 & 67 & 0.2198 & 0.8366 & \\
\hline
\end{tabular}

Table 2: Experimental conditions for ramp up experiments, and transition sequence reported. Samples rheological characterisation is reported in table 1. CF: Couette Flow, TVF: Taylor Vortex Flow, WTVF: Wavy Taylor Vortex Flow, RSW: Rotating Spiral Waves, SVF: Spiral Vortex Flow, EIT: Elasto-Inertial Turbulence.

\begin{tabular}{lllllllll} 
Sample & Test & $f_{s}$ & $\Omega$ & $\mathrm{Re}$ & $\bar{n}_{e}$ & $\overline{\mathrm{El}}$ & $\Gamma_{0}$ & State \\
& & $\mathrm{Hz}$ & $\mathrm{s}^{-1}$ & & & & & \\
\hline $\mathrm{XG200-50}$ & $\mathrm{S} 1$ & 620 & 12.22 & 76 & 0.73 & 0.14 & 0.5078 & $\mathrm{RSW}$ \\
$\mathrm{XG200-50}$ & $\mathrm{S} 2$ & 620 & 16.80 & 114 & 0.73 & 0.14 & 0.5078 & WTVF \\
XG200-50 & $\mathrm{S} 3$ & 620 & 25.96 & 198 & 0.73 & 0.14 & 0.5078 & WTVF \\
XG2000-50 & $\mathrm{S} 1$ & 600 & 26.44 & 52 & 0.47 & 72 & 0.2216 & SVF \\
XG2000-50 & $\mathrm{S} 2$ & 600 & 31.73 & 69 & 0.47 & 72 & 0.2216 & $\mathrm{RSW}$ \\
$\mathrm{XG2000-50}$ & $\mathrm{S} 3$ & 600 & 34.37 & 79 & 0.47 & 72 & 0.2216 & RSW-EIT \\
XG2000-50 & $\mathrm{S} 4$ & 600 & 52.88 & 152 & 0.47 & 72 & 0.2216 & EIT \\
\hline
\end{tabular}

Table 3: Experimental conditions: Steady state experiments. $\Gamma_{0}$ specifies the maximum non-dimensional acceleration rate used in the ramp up leading to the recorded steady state. 
state experiments, the frame rates used allowed all temporal frequencies in the flow to be resolved.

The $2176 \times 16 \mathrm{pixel}^{2}$ images were horizontally averaged to form an axial intensity profile for each instance. All such profiles were compiled into a matrix, or flow map, showing the evolution of the intensity profile over time or any time dependent parameter, such as Re (see e.g figure $6 \mathrm{~b}$ ). In a second step, the flow maps were divided into segments of $N_{c}=256$ columns (successive snapshots), with a $50 \%$ overlap, in order to calculate the average Fast Fourier Transform (FFT) for each row (i.e. each axial location) in each segment. All the averaged spectra were subsequently compiled to form a frequency map (see e.g. figure 6 c, (Cagney \& Balabani 2019b)). This map shows the evolution of the temporal frequencies of the flow as a function of time or Re. On frequency maps, a clear ridge may appear for regions with $f=f_{\Omega} / 2 \pi$ (see figure figure $6 \mathrm{c}$ ), with fainter additional ridges at $f=k \times f_{\Omega} / 2 \pi$. These ridges arise from the ability of the visualisation method to detect the rigid body rotation frequency of the inner cylinder and its harmonics, and should not be interpreted as fluid flow frequencies.

In this work, the focus is on ramp up experiments: $\Omega$ was increased at a constant rate, $\mathrm{d} \Omega / \mathrm{d} t$, up to a maximum rotation speed $\Omega_{\text {max }}$, which led to an increase in the Reynolds number up to $\operatorname{Re}_{\max } \simeq 300$. For shear-thinning fluids, Re was computed using an effective viscosity $\mu(\dot{\gamma})$ derived from Carreau fittings, and thus increases non-linearly with $\Omega$. The flow maps resolution in terms of Reynolds number is quantified by the ratio $1 / \Delta R e$, reported in table 2 , where $\Delta R e$ is the average Reynolds number variation between two successive images. The (Newtonian) non-dimensional acceleration rate is defined as

$$
\Gamma_{0}=\frac{\mathrm{dRe}}{\mathrm{d} t^{*}}=\frac{\rho^{2} r_{i} d^{3}}{\mu^{2}} \frac{\mathrm{d} \Omega}{\mathrm{d} t},
$$

where $t^{*}=t / t_{v}$ is the time divided by the viscous time-scale. This acceleration rate must be kept low (typically $\Gamma_{0}<1$ ) to ensure that flow states are independent of the cylinder acceleration, i.e. that the ramp up can be treated as a quasi-static process (Dutcher \& Muller 2009). In appendix A of the present work, we present an investigation of the effects of acceleration on our experimental conditions, achieved by performing specific experiments in which the acceleration rate was varied over three decades. Based on the results of this investigation, the acceleration rates used for the main set of experiments (section 3) were selected. Values of ramp up parameters are reported in table 2. Values of the Nahme-Griffith number (see definition in supplementary information) were estimated to be of order $10^{-1}$ at most for all working fluids at all shear rates, thus indicating that viscous heating effects are negligible (White \& Muller 2002b,a).

For shear-thinning fluids, El and $\Gamma_{0}$ were not exactly constant during the ramp up process as the viscous time scale depends on the rotation speed in a non-linear fashion (Dutcher \& Muller 2013; Cagney et al. 2020). In a Carreau fluid, $n_{e}$ also varies with the shear rate, which depends on the rotation speed. Thus reference values for each run, taken as the average over the Reynolds range from 20 to $\operatorname{Re}_{\max }$ are also displayed in table 2. Such run-averaged values are noted by overbar symbols (e.g. $\bar{n}_{e}$ or $\left.\overline{\mathrm{El}}\right)$. The reference value for $\Gamma_{0}$ was chosen as the maximum one and simply noted $\Gamma_{0}$ hereafter for the sake of simplicity.

Additionally, some "steady state" experiments were performed in order to visualise specific flow states for longer times. For this type of experiment, the cylinder was first accelerated at $\mathrm{d} \Omega / \mathrm{d} t$ corresponding to a maximum acceleration rate $\Gamma_{0}$ until the targeted rotation speed/Reynolds number was reached, and the acquisition started. Details of such 
experiments are provided in table 3 . The absence of polymer degradation was verified for all fluids by comparing the steady and oscillatory shear rheological characterisation made before and after the flow visualisation experiments. An example of such a comparison between pre-experiment and post-experiment rheological characterisations is available in the supplementary information for the XG2000-50 case (chosen as the most critical in terms of degradation probability, with its high polymer contribution to the viscosity and the strong shear-rate at which it is employed during experiments).

\section{Results}

\subsection{Overview of flow transitions}

An overview of all flow states and ramp up experiments is first presented. Each experiment starts from $\operatorname{Re}=0$ and Re is progressively increased. Flakes align with dominant flow structures, reflecting light more or less intensely depending on their orientation, allowing to capture various transitions to CF, TVF, WTVF, RSW, EIT, and SVF. A time-space diagram, at constant Re, is provided for each flow state in figure 4 a to f. In TVF, SVF or WTVF, the flakes align with the (wavy/spiraling) Taylor vortices, giving rise to a band like structure (figure 4 a) that may be seen oscillating (figure $4 \mathrm{~b}$ ) or travelling 4 e) for WTVF and SVF, respectively. In RSW, the base TVF structure is still visible but additional patterns appear due to the local alignment of the flakes with axial elastic waves $4 \mathrm{c}$ ). In EIT, the random alignment of flakes with a set of various spatial flow structures translates into a chaotic intensity signal and space-time plot (figure $4 \mathrm{~d}$ ). Note that in $\mathrm{CF}$, flakes are all aligned in the azimuthal direction, and the resulting time-space diagram is homogeneously gray (figure $4 \mathrm{f}$ ).

Examples of spatial and temporal FFT (denoted as $\mathrm{FFT}_{s}$ and $\mathrm{FFT}_{t}$ ) of the spacetime plots are shown in figure $4 \mathrm{~g}$ and $\mathrm{h}$, as a function of the spatial wavelength and temporal frequency, respectively. These are computed along vertical and horizontal lines of subplots from figure 4, respectively. The location of the peak(s) in these spectra, if present, can be used to determine the major spatial wavelength(s) $\lambda$ and temporal frequency frequencie(s) of the flow.

Figure 5 a) shows the succession of flow states encountered during several ramp up processes (only one experiment per working fluid is shown for the sake of readability), in a 3D space of elasticity, shear-thinning $\left(1-\bar{n}_{e}\right)$ and reduced Reynolds number $\left(\operatorname{Re} / \operatorname{Re}_{0}\right)$, where $\operatorname{Re}_{0}=107$ is the critical $\operatorname{Re}_{c}^{T V F}$ Reynolds number for the CF to TVF transition for Newtonian fluids in the present setup. A table summarizing all critical Reynolds numbers for flow transitions is available in the supplementary information file. The results of Cagney et al. (2020) have also been rescaled and added to the graph as dashed lines with the same colour code. Figures $5 \mathrm{~b}$ and $5 \mathrm{c}$ are projections of figure $5 \mathrm{a}$ in $(\mathrm{El}, \mathrm{Re})$ and $\left(1-\bar{n}_{e}, \mathrm{Re}\right)$ planes, respectively. The transition patterns are also summarized in table 2 .

No clear trend appears from the 2D projections: several regimes may co-exist in some regions of the plots. For example at $\operatorname{Re}=2 \times \mathrm{Re}_{0}$ and $\mathrm{El} \sim 0.1$, the P500-72 experiment features EIT while the XG200-50 experiment is in the WTVF state for similar inertia and elasticity (figure $5 \mathrm{~b}$ ). Similarly, at $\mathrm{Re}=2 \times \operatorname{Re}_{0}$ and for $1-n_{e} \sim 0.3$, XG1000-72 experiments feature EIT while XG200-50 is in the WTVF state, while at equivalent levels of inertia and shear-thinning(figure $5 \mathrm{c}$ ). It is therefore necessary to consider all three parameters to distinguish preferential regions for each flow state in the three dimensional parameter space, as done in figure 5 a). WTVF can be found at high $R e / \mathrm{Re}_{0}$, but only for intermediate elasticity. Conversely, EIT requires either strong elasticity or negligible 


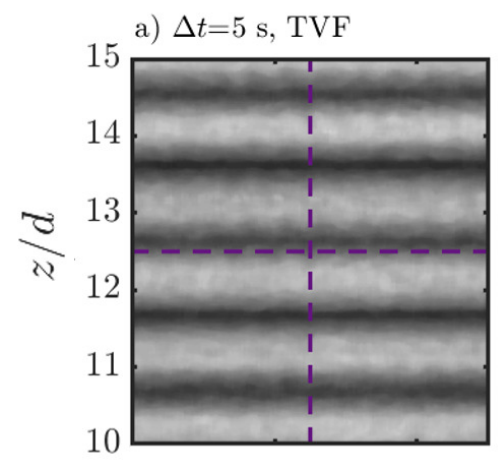

b) $\Delta t=3 \mathrm{~s}$, WTVF

c) $\Delta t=5 \mathrm{~s}, \mathrm{RSW}$
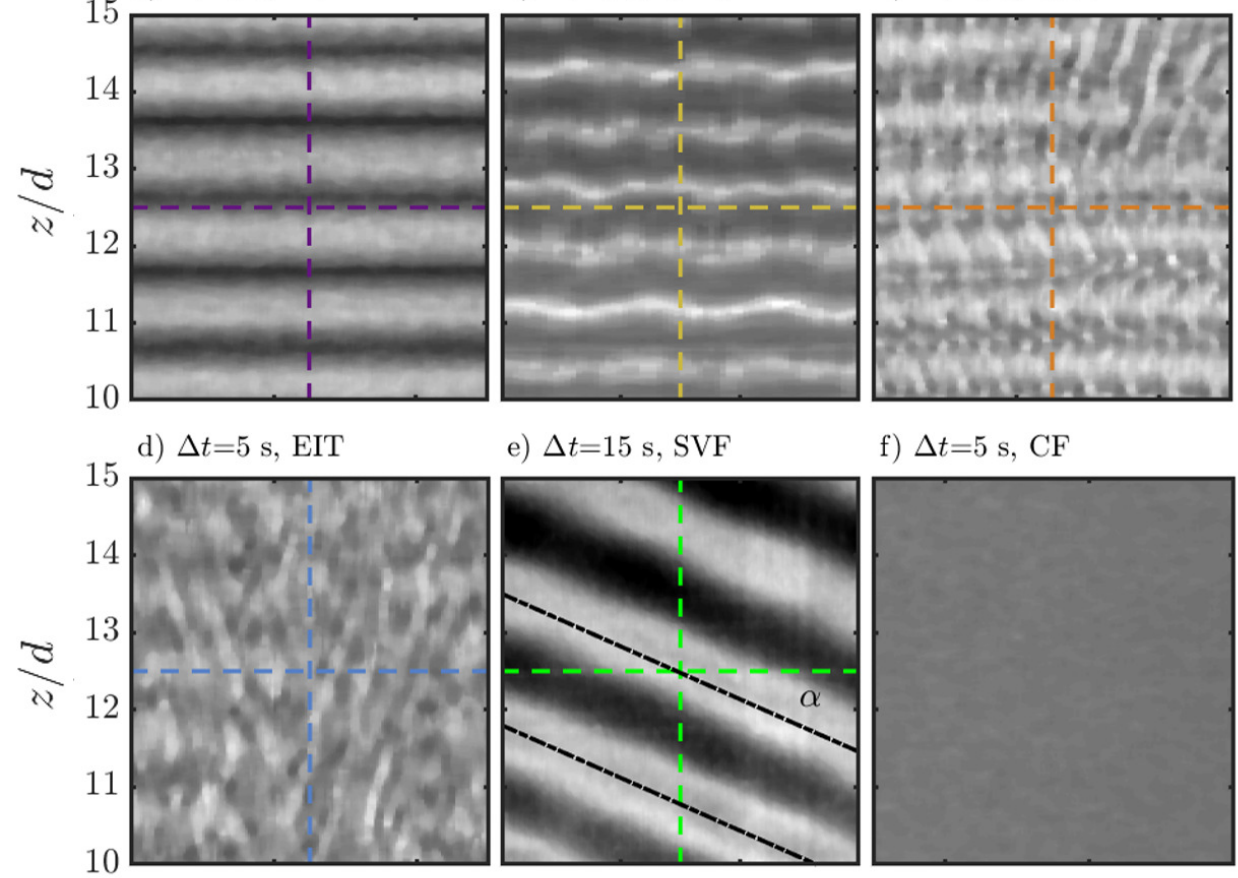

e) $\Delta t=15 \mathrm{~s}, \mathrm{SVF}$

f) $\Delta t=5 \mathrm{~s}, \mathrm{CF}$
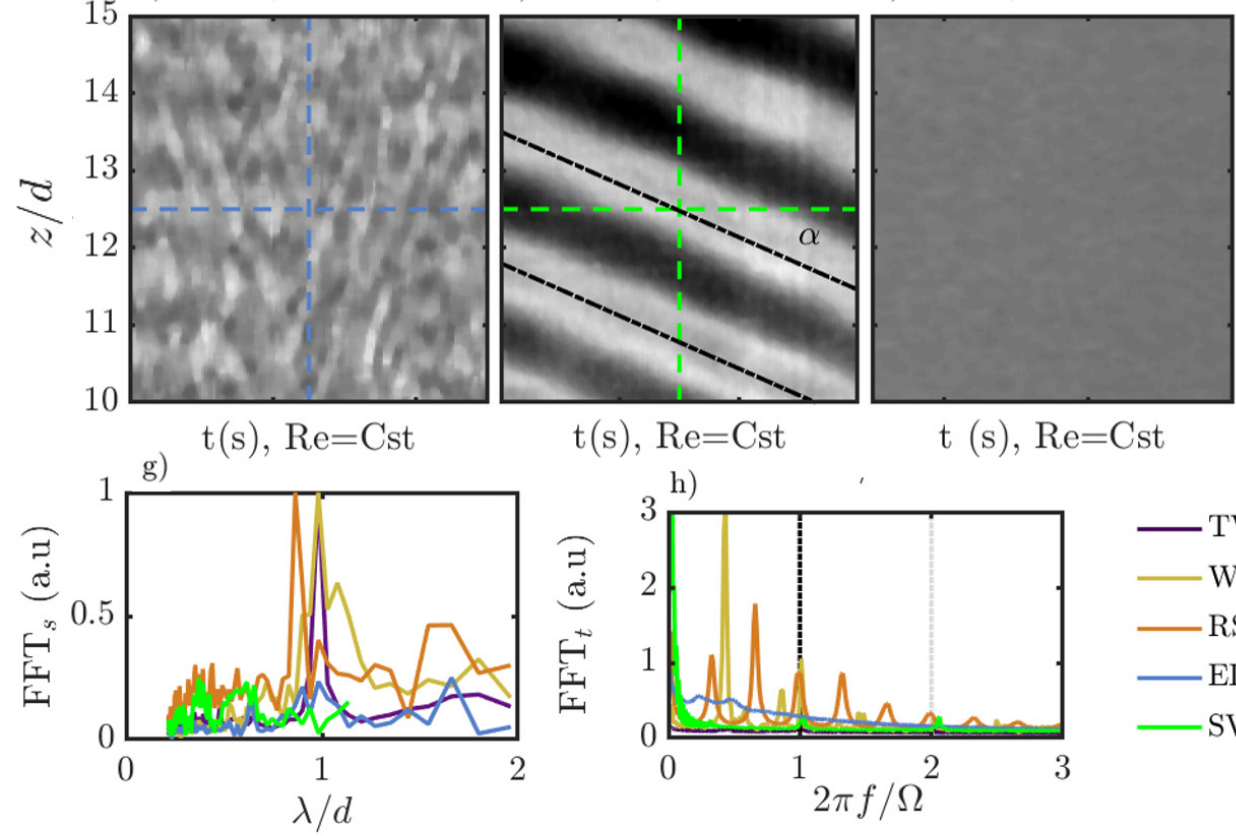

$$
\mathrm{t}(\mathrm{s}), \mathrm{Re}=\mathrm{Cst}
$$

$\mathrm{t}(\mathrm{s}), \mathrm{Re}=\mathrm{Cst}$

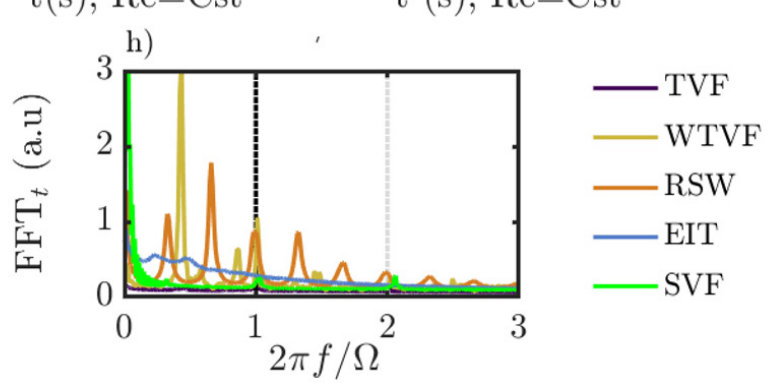

Figure 4: Illustrative panel of observed flow maps and flow states. (a-f) are time-space diagrams on $\Delta t$ intervals of 3 to $15 \mathrm{~s}$ spanning the $10<z / d<15$ vertical region. a) $\mathrm{N} 72, \mathrm{Re}=120 \mathrm{~b}) \mathrm{N} 72, \mathrm{Re}=250$ c) P500-72, Re=110 d) P500-72, Re=150 e) XG2000-50, $\mathrm{Re}=52 \mathrm{f}) \mathrm{P} 500-72, \mathrm{Re}=50 \mathrm{~g})$ and $\mathrm{h}$ ) illustrate typical spatial and temporal spectra,

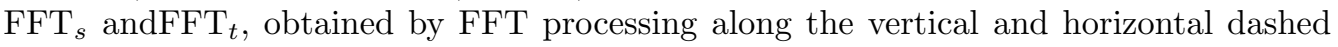
lines in (a-e), respectively.

shear-thinning to develop. The existing conditions of different flow states based on the rheological and inertial parameters are detailed and discussed in the rest of section 3 .

\subsection{Non shear-thinning cases}

\subsubsection{Newtonian fluid}

In the Newtonian reference case, the expected CF-TVF-WTVF transition pattern is retrieved, as illustrated in figure 6 with the N72-1 experiment. Prior to the onset of any 

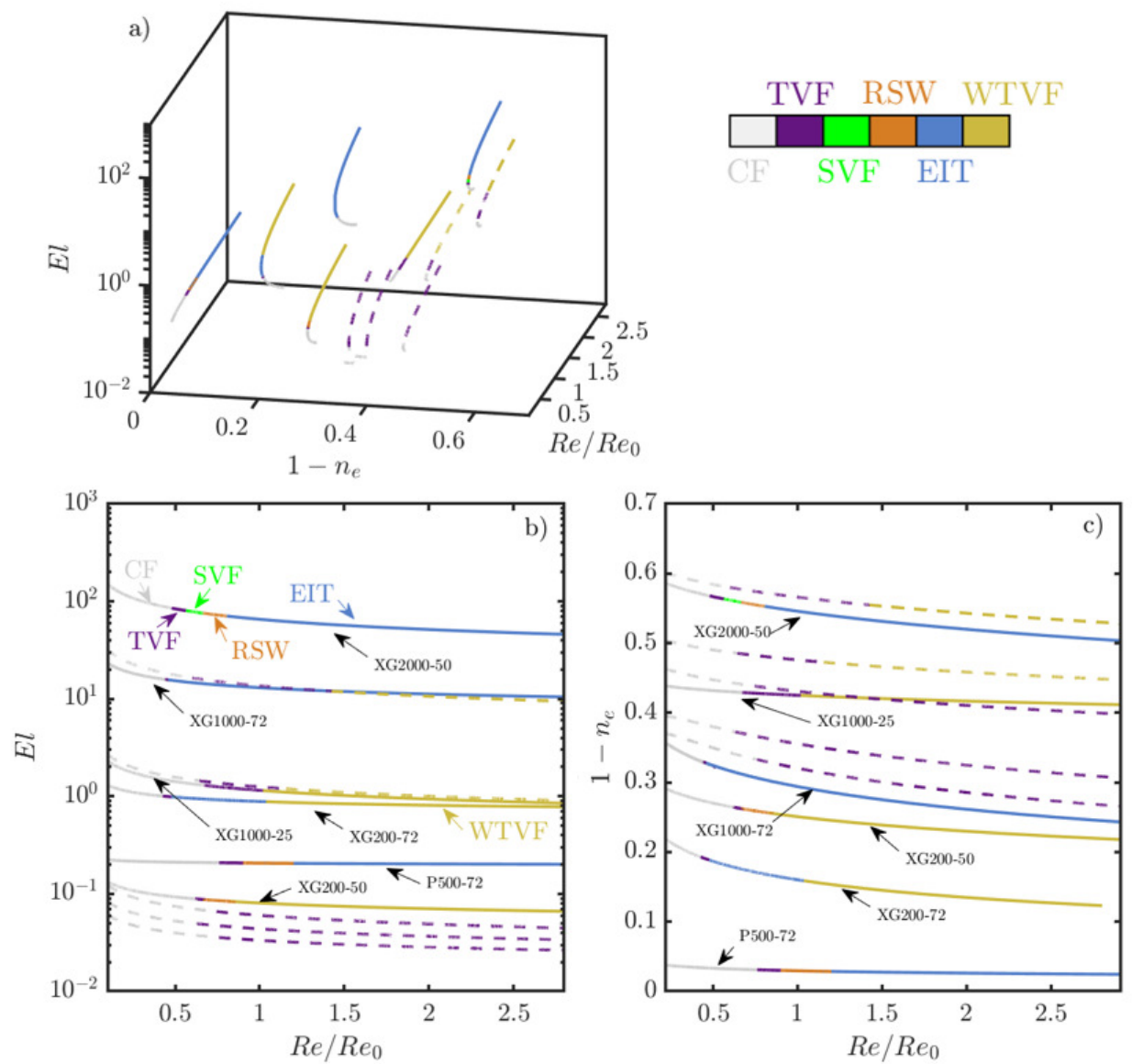

Figure 5: Summary of experiments in $\left(\mathrm{Re} / \mathrm{Re}_{0}, 1-n_{e}, E l\right)(\mathrm{a}),\left(\mathrm{Re} / \mathrm{Re}_{0}, E l\right)$ (b) and $\left(\operatorname{Re} / \operatorname{Re}_{0}, 1-n_{e}\right)$ (c) parameter spaces. Each path corresponds to a single ramp up experiment. Each flow state is associated with a line colour according to the legend (colours online). Full lines report experiments from the present work, and dashed lines experiments from Cagney et al. (2020), performed in similar conditions (polymer batch, geometry, ramp up protocol, flow visualisation method) on xanthan gum samples at various concentrations dissolved in a $25 \%$ glycerol in water solvent.

instability, Ekman vortices (Cole 1976) develop at the top and bottom of the cylinder (see e.g. figure $6 \mathrm{~b}$ ), top and bottom). The transition from CF to a band-like TVF structure can be clearly seen by a jump in the rms value of the z-intensity profile 6 a, denoted $i^{*}$. The CF to TVF transition occurs at $\operatorname{Re}_{c}^{T V F}=107=\operatorname{Re}_{0}$ and the transition from TVF to WTVF occurs at $\operatorname{Re}_{c}^{W T V F}=218=2.03 \times \operatorname{Re}_{0}$. The latter, while difficult to observe on the flow map (figure $6 \mathrm{~b}$ ) due to a weak spatial amplitude, is clearly seen on the frequency map $(6 \mathrm{c})$ when an additional frequency ridge appears. Both results are consistent with the values reported in the literature (see for example Dutcher \& Muller (2009); Cagney \& Balabani (2019b); Ramesh et al. (2019)), ranging from 80 to 120 and 170 to 450 respectively. The wavelength of TVF is $\lambda=0.89 \mathrm{~d}$. The critical wavy frequency at the onset of the WTVF is $f_{w}=1.02 \times f_{\max }=1.40 \times \frac{\Omega}{2 \pi}$. 


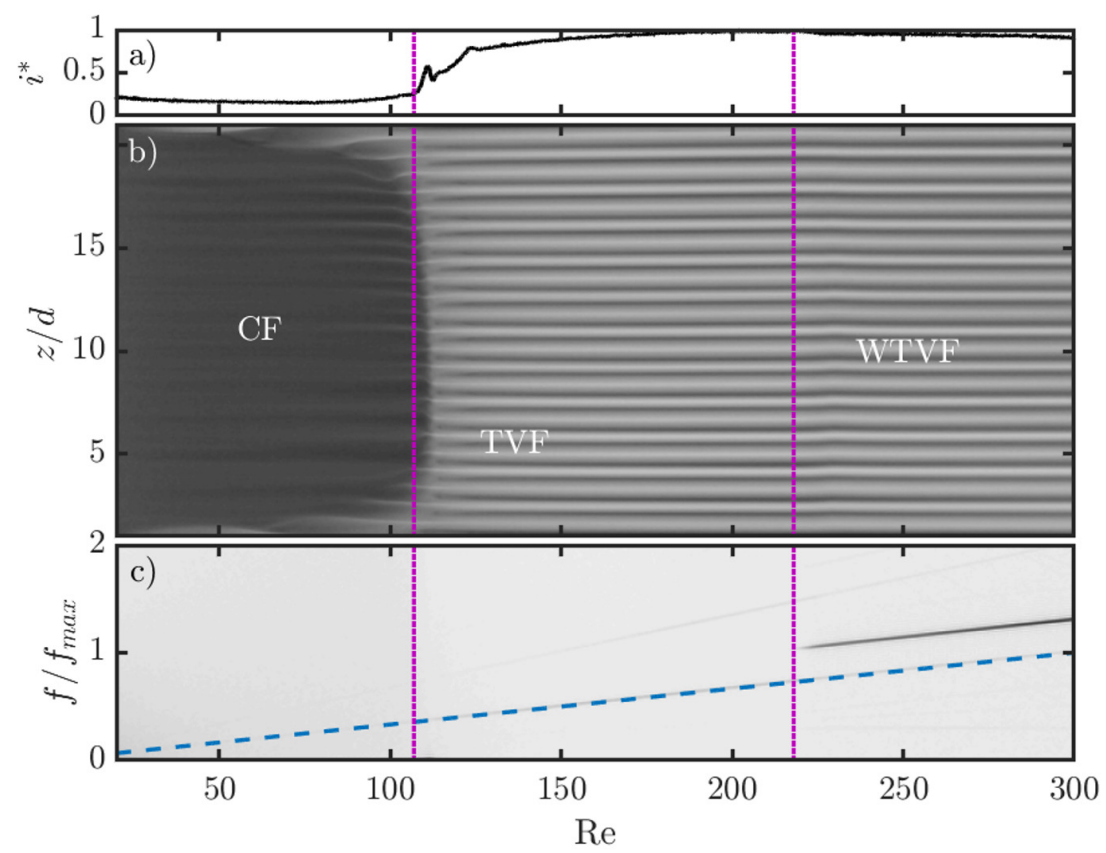

Figure 6: Intensity signal (a), flow map (b) and frequency map (c) for the N72-1 experiment $\left(\overline{\mathrm{El}}=0, \bar{n}_{e}=1\right)$. In a) and all subsequent similar figures, $i^{*}$ is the root mean square gray level intensity of sub-plot b) along the $z / d$ dimension, scaled by its maximum value on the Re range. The dashed line on sub-figure $\mathrm{c}$ denotes the inner cylinder rotation frequency $f_{\Omega} \cdot f_{m} a x$ is the rotation frequency corresponding to $\left.\mathcal{R}\right\rceil_{m} a x$. Vertical dotted lines shows the transitions between flow regimes.

\subsubsection{Boger fluid}

In the Boger fluid case (P500-72), the transition pattern observed is CF-TVF-RSWEIT (see figure 7). It is consistent with transitions reported in the literature for moderate or high elasticity fluids (Groisman \& Steinberg 1996; Schaefer et al. 2018). The onset of TVF happens at lower Re than in the Newtonian case, $\operatorname{Re}_{c}^{T V F}=97=0.90 \times \mathrm{Re}_{0}$. Elastic waves (RSW) appear soon and abruptly after the onset of the TVF state, at $\operatorname{Re}_{c}^{R S W}=$ $103=0.96 \times \mathrm{Re}_{0}$, as several horizontal ridges become visible in the frequency map (figure $7 \mathrm{c}$ ). These correspond to elastic waves, the frequency of which is not correlated to the Reynolds number but rather to the constant elastic number, through the elastic time scale (see Gillissen (2019); Lacassagne et al. (2020)). As a point of comparison, Dutcher \& Muller (2013) reports transition to TVF at $\operatorname{Re}_{c}^{T V F} / \operatorname{Re}_{0}=1.07$ and to $\mathrm{RSW}$ at $\operatorname{Re}_{c}^{R S W} / \operatorname{Re}_{c}^{0}=1.13$ in moderately elastic Boger fluids $(E l=0.1-0.2)$. The apparent spatial wavelengths are $\lambda=0.82 d$ and $\lambda=0.89 d$ in TVF and RSW states, respectively, while Dutcher \& Muller $(2013)$ reported $\lambda \approx 1.6 d$ in both states. The flow finally becomes increasingly disordered with Re, and quickly transitions to EIT at Re values for which it would be laminar under purely inertial conditions.

\subsection{Shear-thinning dominated fluids}

In the highly shear-thinning $\left(\bar{n}_{e}=0.5766\right)$ XG1000-25-1 case, a Newtonian-like transition pattern is observed: CF-TVF-WTVF (figure 8). A similar behaviour has been reported in several other studies where the fluid exhibited a non-negligible shear-thinning 


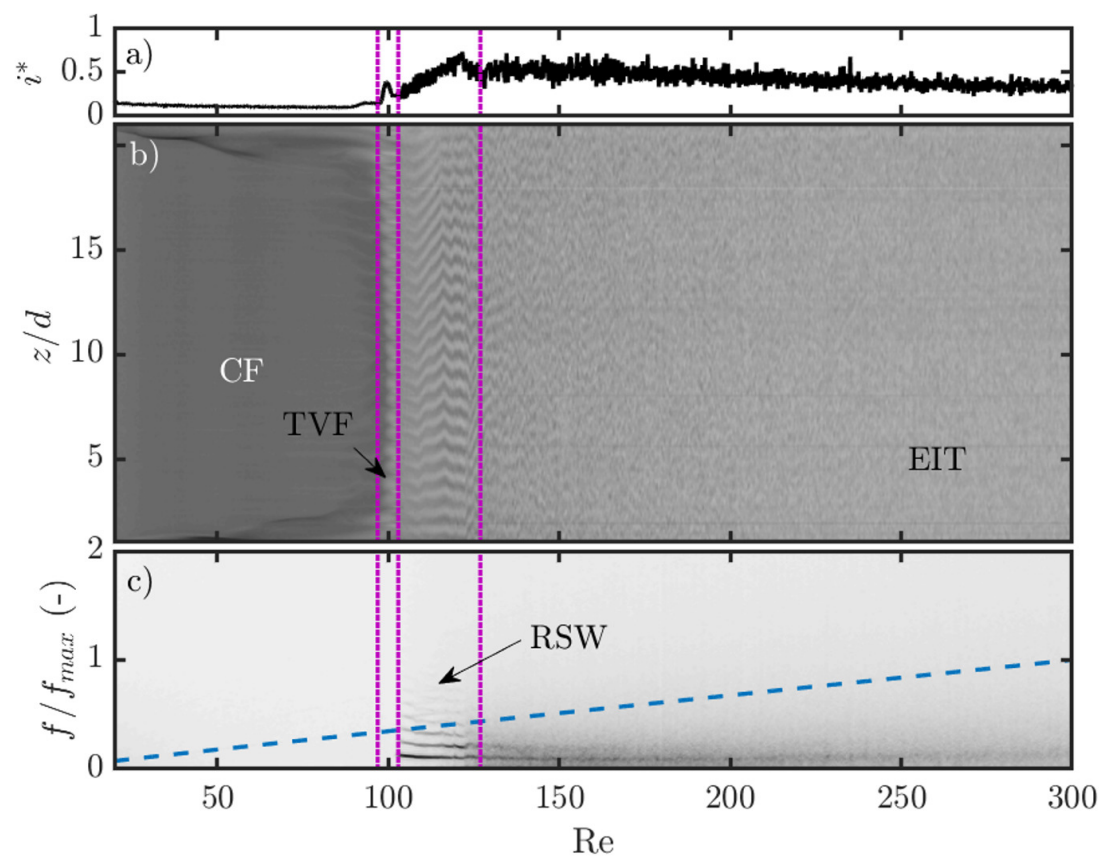

Figure 7: Intensity signal (a), flow map (b) and frequency map (c) for the P500-723 experiment $\left(\overline{\mathrm{El}}=0.19, \bar{n}_{e}=0.96\right)$. The dashed line on sub-figure $\mathrm{c}$ denotes the inner cylinder rotation frequency. Vertical dotted lines denote the transitions between flow regimes.

behaviour and negligible to moderate elasticity (Crumeyrolle et al. 2002; Cagney \& Balabani 2019b,a; Cagney et al. 2020). Here it occurs despite the elastic number $(\overline{\mathrm{El}}=$ 1.2) being approximately five times that of the fluid discussed in the previous section. Boger fluids of equivalent elastic number in the present experimental set-up are expected to exhibit elastic flow features such as RSW or transition to EIT, as suggested by experiment reported above (section 3.2.2). The absence of these in the flow map of figure 8 suggests that elastic effects are suppressed by shear-thinning. The CF to TVF transition occurs at $\operatorname{Re}_{c}^{T V F}=72=0.67 \times \mathrm{Re}_{0}$. The flow is thus destabilised by combined shear-thinning and elasticity. The spatial wavelength of TVF is $\lambda=0.99$, which is, as expected, slightly larger than that for the Newtonian case (Escudier et al. 1995; Cagney $\&$ Balabani 2019b). The WTVF arises at $\operatorname{Re}_{c}^{W T V F}=109=1.01 \times \mathrm{Re}_{0}$, again much sooner than in the Newtonian case. Moreover, at the onset of WTVF, the wave frequency is lower than the inner rotation frequency $\left(f_{w}=0.432 \times \frac{\Omega}{2 \pi}\right)$ while it is higher in the Newtonian case. Finally, the complexity of the WTVF is enhanced, with ridges appearing and disappearing as Re increases.

\subsection{Elasticity dominated fluids}

By keeping the same polymer at the same concentration (XG $1000 \mathrm{ppm}$ ) but simply increasing the solvent viscosity (XG1000-72-2 experiment), it is possible to produce a fluid of much higher elasticity $(\overline{\mathrm{El}}=13)$ and weaker shear-thinning $\left(\bar{n}_{e}=0.71\right)$. This fluid exhibits strikingly different patterns (figure 9). The flow abruptly transitions from CF to EIT, which is the typical behaviour of highly elastic fluids (Groisman \& Steinberg 1996). This transition is easily detected from the abrupt jump in intensity rms signal 


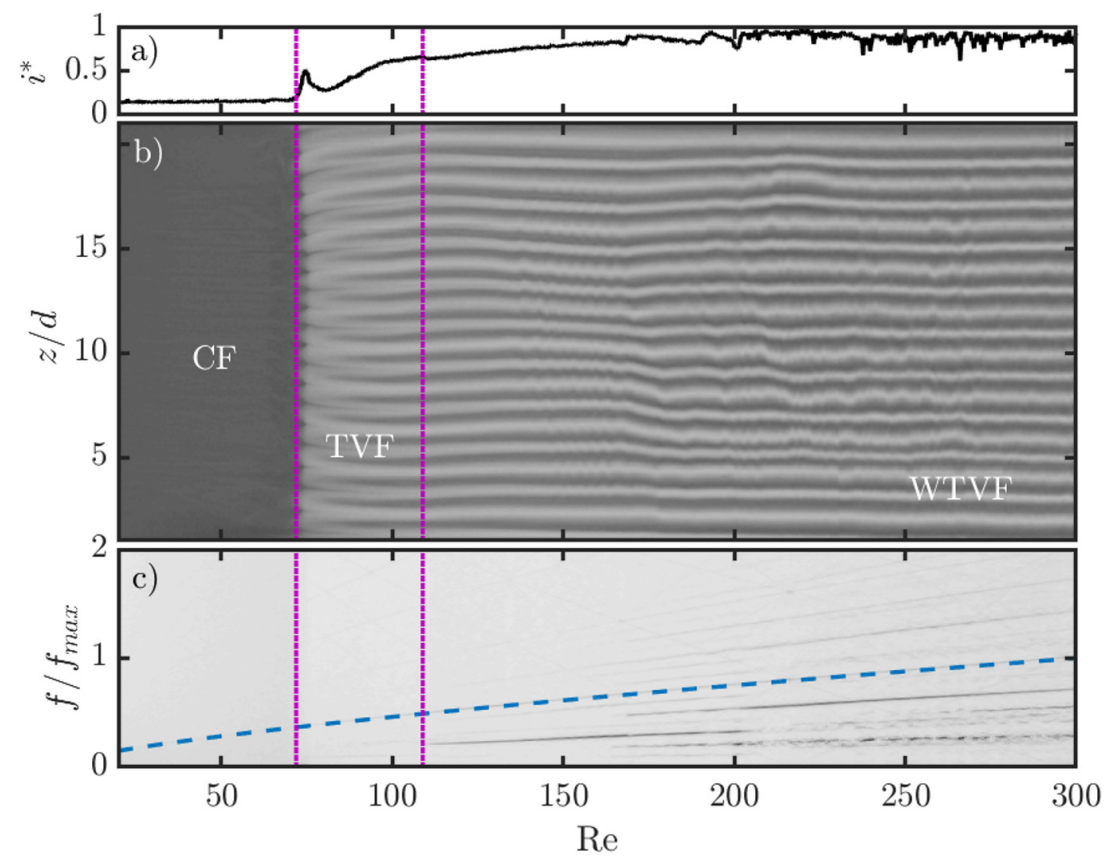

Figure 8: Intensity signal (a), flow map (b) and frequency map (c) for the XG100025-1 experiment $\left(\overline{\mathrm{El}}=1.2, \bar{n}_{e}=0.58\right)$. The dashed line on sub-figure c denotes the inner cylinder rotation frequency. Vertical dotted lines denote the transitions between flow regimes. Note that the rotation frequency detected varies non-linearly with Re, due to the shear-thinning behaviour (Cagney \& Balabani 2019b).

(figure 9 a). No specific frequency can be identified in the flow map, as was also the case in the Boger fluid (figure 7) in the EIT state. The CF-EIT transition occurs at $\operatorname{Re}_{c}^{E I T}=47=0.44 \times \mathrm{Re}_{0}$, which is in line with the results reported by Groisman \& Steinberg (1996): elasticity strongly destabilizes the base Couette flow. While the latter is a well known result, the very different transition sequences in figures 8 and 9 constitute an interesting novelty of this study. Depending on the relative degrees of shear-thinning and elasticity dictated by the solvent properties, the flow of the same polymer at the same concentration may exhibit completely different hydrodynamic behaviours. Observations thus suggest that not only elasticity destabilises the flow, but it can also induce various hydrodynamic regimes by tuning the relative degrees of shear thinning and elasticity.

\subsection{Moderate shear-thinning and elasticity}

Through changes in both solvent viscosity and polymer concentration, we can then keep a similar flow index $\left(\bar{n}_{e} \approx 0.8\right)$ and adjust the elasticity to be equivalent to that in the Boger fluid $(\overline{\mathrm{El}} \approx 0.1$, figure 7 , sub-section 3.2 .2$)$ ), in order to probe the flow transitions in the moderate elasticity, moderate shear thinning regime of the parameter space (figure 5).

The behaviour (illustrated in figure 10, XG200-50-3 ramp up experiment) approaches that of a fluid dominated by shear-thinning, with a Newtonian-like transition pattern, as shown in figure 8. The only notable difference is the existence of an intermediate RSW state between the TVF and WTVF states. The CF to TVF transition occurs at $\operatorname{Re}_{c}^{T V F} / \operatorname{Re}_{0}=0.62$. RSW waves appear at $\operatorname{Re}_{c}^{R S W} / \operatorname{Re}_{0}=0.67$ and completely disappear 


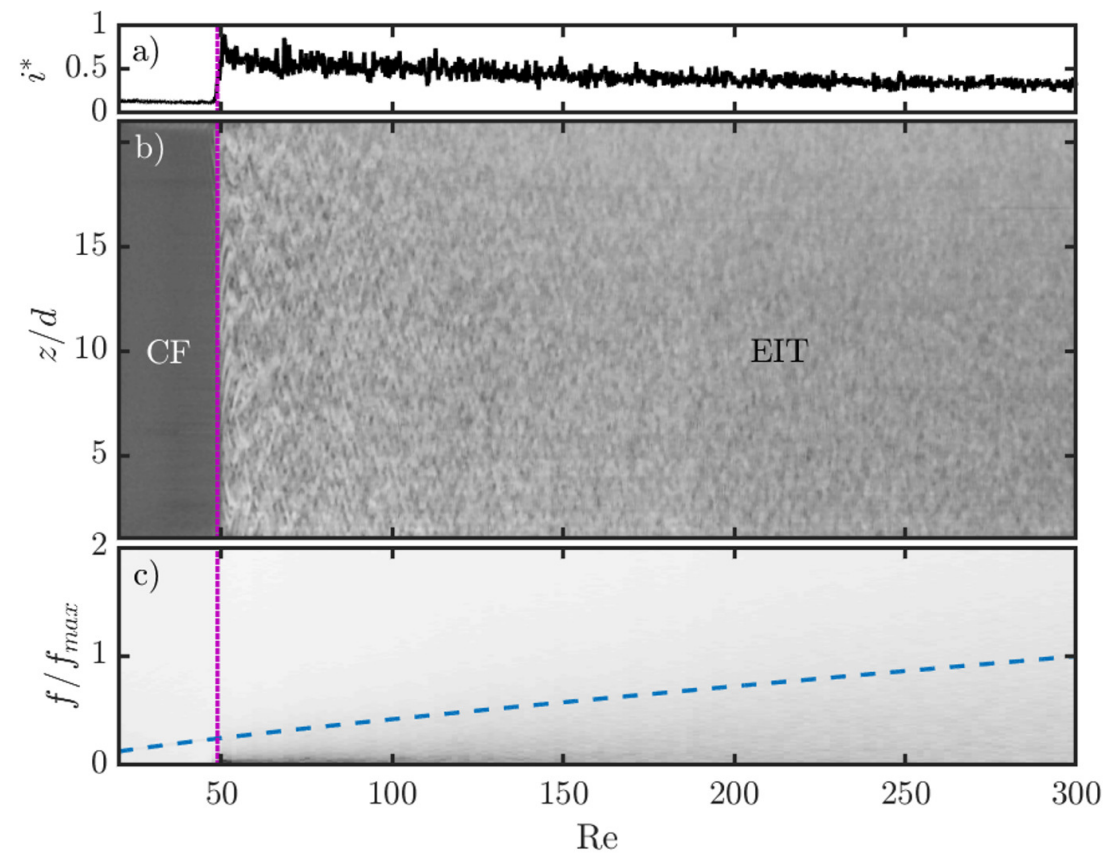

Figure 9: Intensity signal (a), flow map (b) and frequency map (c) for the XG1000-72-2 experiment $\left(\overline{\mathrm{El}}=13, \bar{n}_{e}=0.71\right)$. The dashed line on sub-figure c denotes the inner cylinder rotation frequency. Vertical dotted lines denote the transition from CF to EIT. Note that the rotation frequency detected varies non-linearly with Re, due to the shear-thinning behaviour, but this non-linear trend is weaker than in figure $8 \mathrm{c}$ ), the shear-thinning index being here closer to 1 .

after $\operatorname{Re}_{c}^{W T V F} / \operatorname{Re}_{0}=0.83$. This RSW pattern bears some similarities with that exhibited by the Boger fluid (figure 7). However, shear-thinning seems to modify the nature of the RSW waves by enhancing their dependency on inertia, since the ridges are not completely Re-independent (figure $10 \mathrm{c}$ ).

Instead of EIT (as occurs for a Boger or elasticity dominated fluid), the flow transitions back to WTVF with increasing Re. The WTVF state exists at Re values lower than in the Newtonian case $(\mathrm{Re} \simeq 100)$, in line with the results for Newtonian-like shearthinning fluids described in section 3.3. In order to investigate this specific RSW to WTVF transition further, steady state experiments were performed on this fluid at three different Re values: $\mathrm{Re}=76$ (S1), $\mathrm{Re}=114$ (S2) and $\mathrm{Re}=198$ (S3) (see table 3). These are described in figure 11.

The S1 case (figure 11 a and d) displays a RSW pattern in which the base TVF structure is still visible, but periodic patterns are very pronounced. The frequency spectrum (figure $11 \mathrm{~h}$ ) clearly shows multiple peaks, with the highest energy peak found at $f=0.33 \times \frac{\Omega}{2 \pi}$. The $\mathrm{S} 2$ case (figure $11 \mathrm{~b}$ and e) lies between S1 and S3, with an evident WTVF structure; however, darker and whiter spots can be detected along given horizontal lines (figure 11 e), indicating persisting RSW waves. The S3 case (figure 11 c and $\mathrm{f}$ ) shows a WTVF pattern similar to other reported shear-thinning WTVF states (figure 8, Cagney \& Balabani $(2019 b)$ ). The wavy frequency is lower than the inner cylinder frequency $\left(f_{w}=0.750 \times \frac{\Omega}{2 \pi}\right.$ at $\operatorname{Re}=198$, or $f_{w}=0.689 \times \frac{\Omega}{2 \pi}$ at $\operatorname{Re}=150$ in figure $10 \mathrm{c}$ ). Multiple secondary peaks are visible, indicating a complex wavy behaviour. 


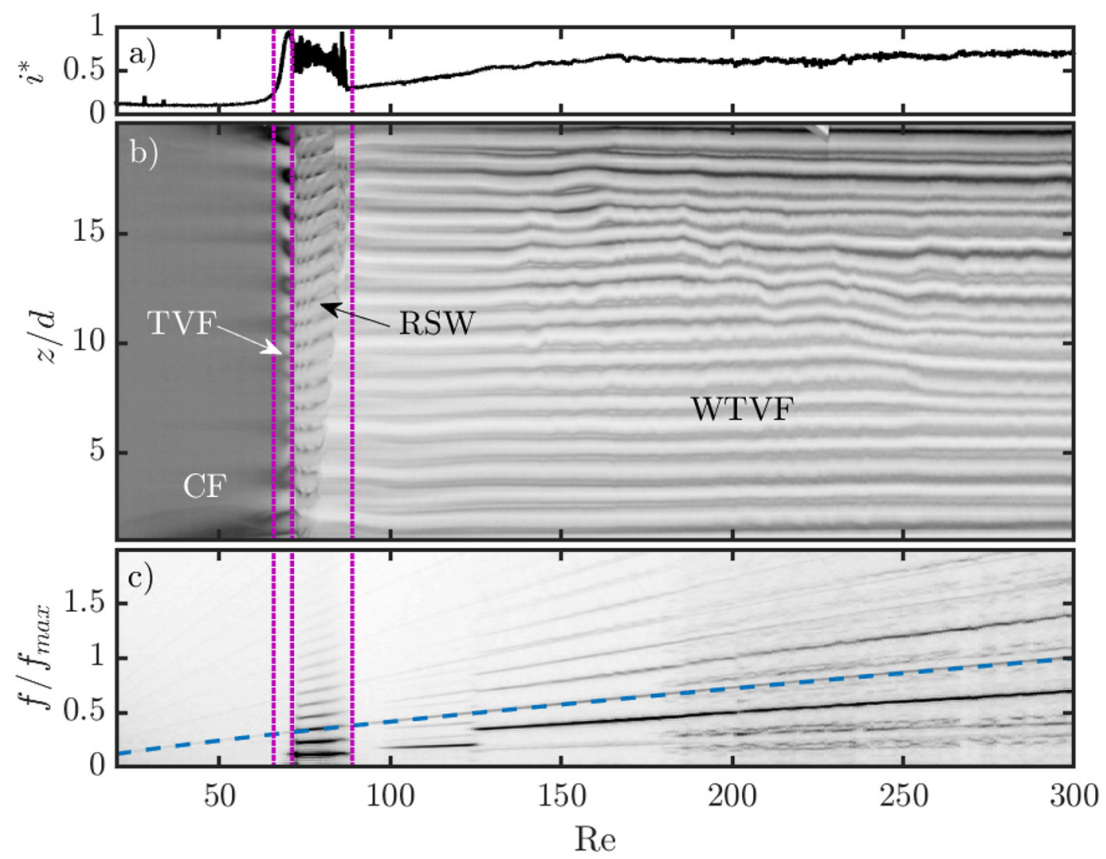

Figure 10: Intensity signal (a), flow map (b) and frequency map (c) for the XG20050-3 experiment $\left(\overline{\mathrm{El}}=0.14, \bar{n}_{e}=0.73\right)$. The dashed line on sub-figure $\mathrm{c}$ denotes the inner cylinder rotation frequency. Vertical dotted lines denote the transitions between flow states. Note that the rotation frequency detected varies non-linearly with Re, due to the shear-thinning behaviour (Cagney \& Balabani 2019b).

S2 and S3 share a common peak at the same $f_{w}$ value when the frequency is scaled by $\Omega$ (figure $11 \mathrm{~h}$ ). This peak is thus characteristic of an inertial flow feature, namely, WTVF. On the other hand, the lower frequency peak found in S1 at $f=0.33 \times \frac{\Omega}{2 \pi}$ is retrieved on S2 and S3 but progressively drifts towards higher frequencies with increasing Re when the same scaling is used. This suggests that this other peak is not associated with inertia, but rather correlates with the elastic wave frequency (see figure 7, Lacassagne et al. (2020)). This elastic feature $\left(f_{w}\right.$ scaling with $\left.t_{e}^{-1}\right)$ progressively yields to inertia $\left(f_{w}\right.$ scaling with $\Omega$ ), as the flow gradually transitions from RSW to WTVF. The fact that the RSW ridges are not perfectly horizontal in the frequency maps suggests that, in shear-thinning fluids, both inertial and elastic effects are involved in the RSW pattern.

Further insight is brought by slightly shifting the fluids properties towards a more elastic, less shear-tinning case (XG200-72, $\left.\overline{\mathrm{El}}=0.88, \bar{n}_{e}=0.85\right)$. The transition pattern is then CF-TVF-RSW-EIT-WTVF, with an additional EIT state appearing between RSW and WTVF. The CF to TVF transition occurs at $\mathrm{Re}_{c}^{T V F} / \mathrm{Re}_{0}=0.43$. RSW develops over a very limited $\mathrm{Re}$ range, from $\operatorname{Re}_{c}^{R S W} / \operatorname{Re}_{0}=0.48$, quickly transitioning to EIT, and then smoothly to WTVF at $\operatorname{Re}_{c}^{W T V F} / \operatorname{Re}_{0}=1.0$. At $\operatorname{Re}=150=1.4 \times \operatorname{Re}_{0}$ where the wavy frequency ridge is clearly visible on the frequency map (figure $12 \mathrm{c}$ ), the wavy frequency is $f_{w}=0.451 \times \frac{\Omega}{2 \pi}$.

This other fluid thus displays an elastic transition sequence(CF-TVF-RSW-EIT) until a sufficient amount of inertia (Re) is reached, and the flow re-transitions to a Newtonianlike behaviour, yet marked by shear-thinning. It is thus an extension of the previous case (XG200-50, figure 10) where elasticity plays a greater role in determining the nature 

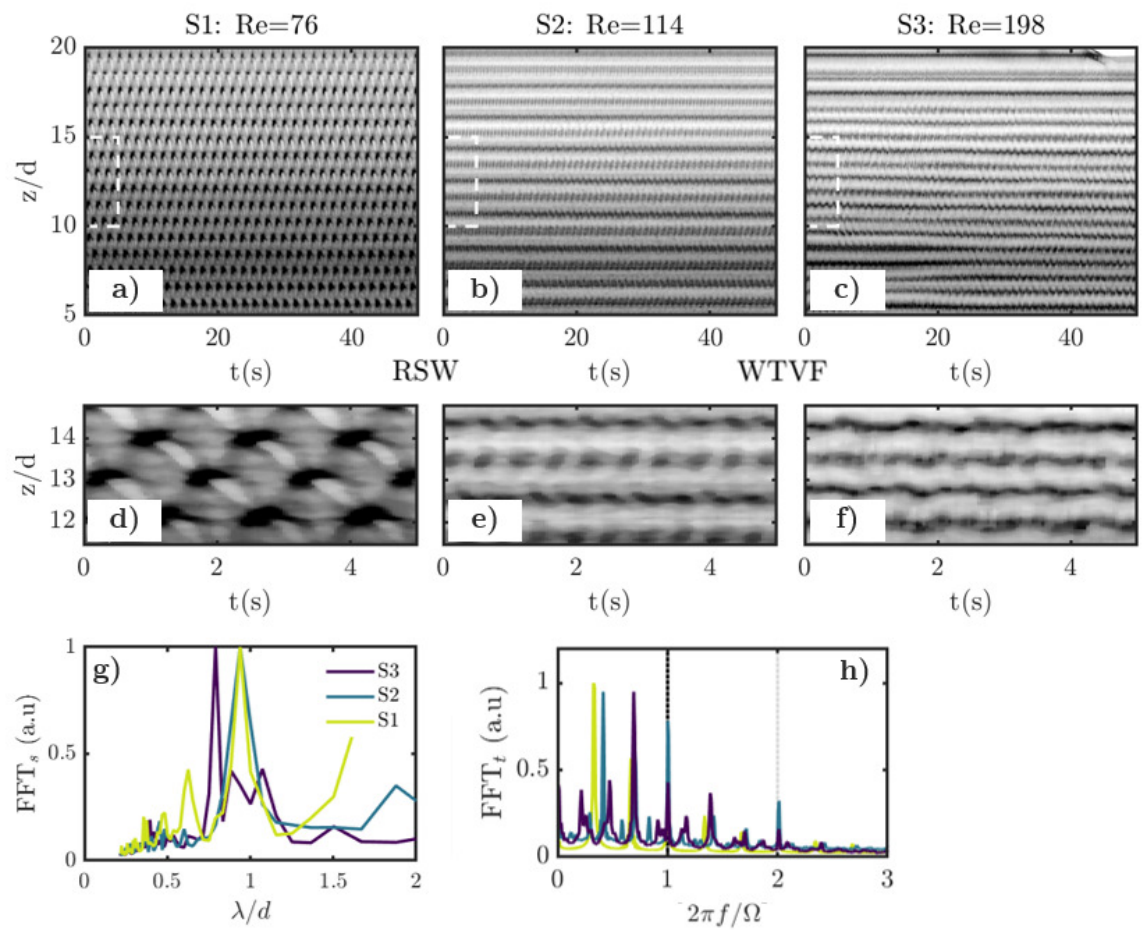

Figure 11: Steady state recordings at constant Re of successive RIB and WTVF states for the XG200-50 case $\left(\overline{\mathrm{El}}=0.14, \bar{n}_{e}=0.73\right)$. (a-c) Time-space diagrams over $50 \mathrm{~s}$ time spans. (d-f) Close-ups of the same flow maps on the first 5 seconds and in the central region (dashed rectangles). g) Spatial spectrum and h) temporal spectrum of the three steady state experiments.

of the transitions, as expected from the variations in rheological properties between the two fluids. It also leads to a counter-intuitive observation: the complexity of the flow and degree of chaos, induced by elasticity, can be reduced when increasing inertia together with shear-thinning. This intriguing behaviour cannot be attributed to polymer degradation, as rheological characterisation before and after confirmed the integrity of the polymer solutions. A possible explanation is the existence of an underlying damping mechanism of elastic waves with increasing inertia (Re).

Shear-thinning may compete with elasticity to condition the existence of elastic waves of flow patterns in the fluid (as previously observed by comparing figures 8 and 9), even to the point of re-laminarising the flow (figures 10, 12). Mild shear-thinning with moderate inertia allows elastic waves to exist, but high shear-thinning with moderate inertia (see XG1000-25 case), or mild shear-thinning with higher inertia (XG200-50, XG200-72) do not.

\subsection{Highly shear-thinning, highly elastic fluids}

Experiments with a highly elastic and shear-thinning fluid (XG2000-50-2, $\overline{\mathrm{El}}=74$, $\left.\bar{n}_{e}=0.56\right)$ are reported in figure 13 . The flow transition pattern is mostly elastic-like, with the final state being EIT (for Re $<300$ ). It is yet different from that of a Boger fluid, with an additional SVF (Spiral Vortex Flow) pattern appearing between TVF and 


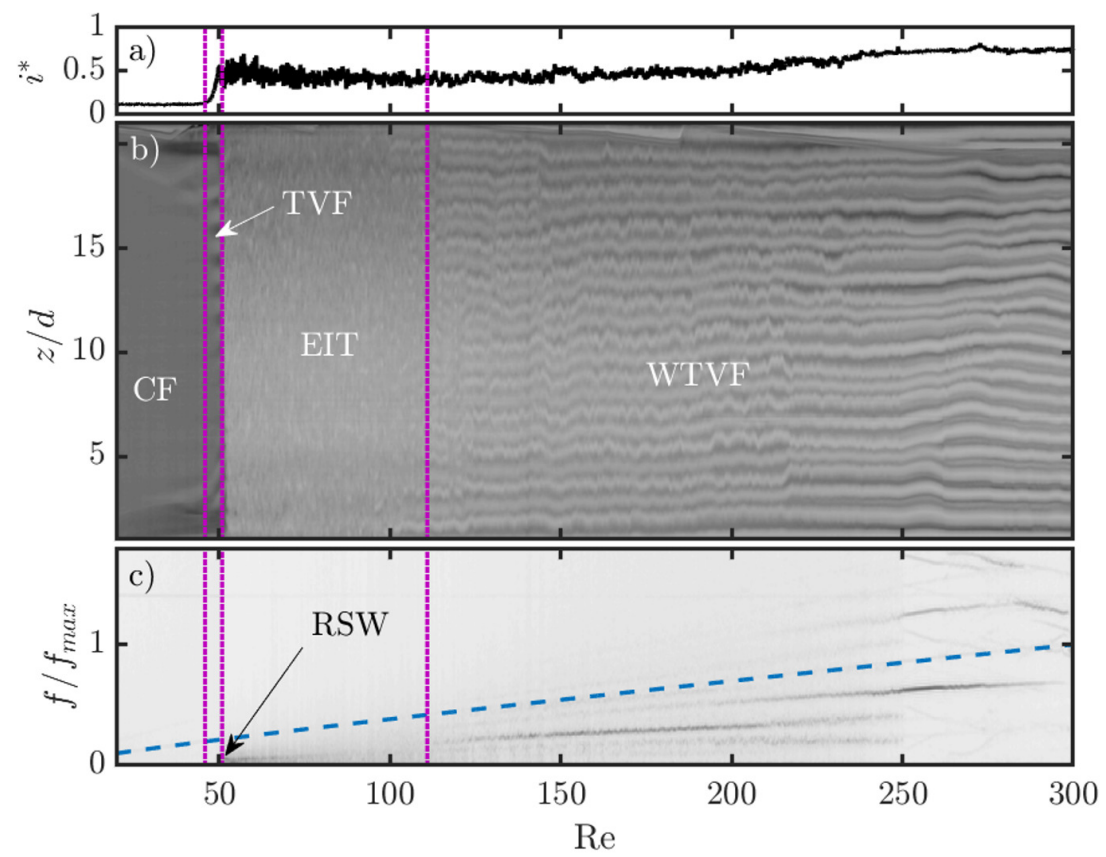

Figure 12: Intensity signal (a), flow map (b) and frequency map (c) for the XG200$72-1$ experiment $\left(\overline{\mathrm{El}}=0.88, \bar{n}_{e}=0.85\right)$. The dashed line on sub-figure $\mathrm{c}$ denotes the inner cylinder rotation frequency. Vertical dotted lines denote the transitions between flow states. Note that the rotation frequency detected varies non-linearly with Re, due to the shear-thinning behaviour (Cagney \& Balabani 2019b).

RSW. The transition sequence is thus CF-TVF-SVF-RSW-EIT. As expected from the strong elasticity and shear-thinning properties of the fluid, the CF destabilises at low Re values, and the onset of TVF occurs at $\operatorname{Re}_{c}^{T V F}=37=0.34 \times \operatorname{Re}_{0}$. EIT is reached at $\operatorname{Re}_{c}^{E I T}=78=0.73 \times \operatorname{Re}_{0}$. The intermediate flow states therefore occur within a short Reynolds span. The transitions to RSW and EIT are close in Re values, which compares well to the behaviour observed previously in the XG200-72 experiment (section 3.5, figure 12).

The additional SVF flow state corresponds to Taylor vortices spiralling and travelling along the axial direction, which translates on the flow maps as slanted dark and white stripes, not showing any particular temporal signature on the frequency map. The existence of such a flow state has been reported in elastic fluids (including the experiments of Baumert \& Muller (1997), linear stability analysis of Avgousti \& Beris (1993)), solid particle suspensions (Ramesh et al. 2019; Ramesh \& Alam 2020), but also Newtonian fluids with counter-rotating outer cylinder (Andereck et al. 1986). Sudden changes of the spiralling propagation direction may occur and give rise to a similar flow state called the Inter-penetrating Spiral Vortex Flow (ISVF) (Ramesh \& Alam 2020; Baumert \& Muller 1997). In the recent works by Elçiçek \& Güzel (2020b,a) SVF (sometimes coexisting with TVF) has also been observed in shear-thinning (possibly also elastic) XG solutions. Remarkably, SVF and ISVF have been found to coexist with RIB states under certain conditions (Ramesh \& Alam 2020). Indeed, RIB is actually the flow pattern resulting from the interactions between up-going and down-going stationary spirals of similar amplitudes. Latrache and co-workers (Latrache et al. 2016) have shown that in shear- 


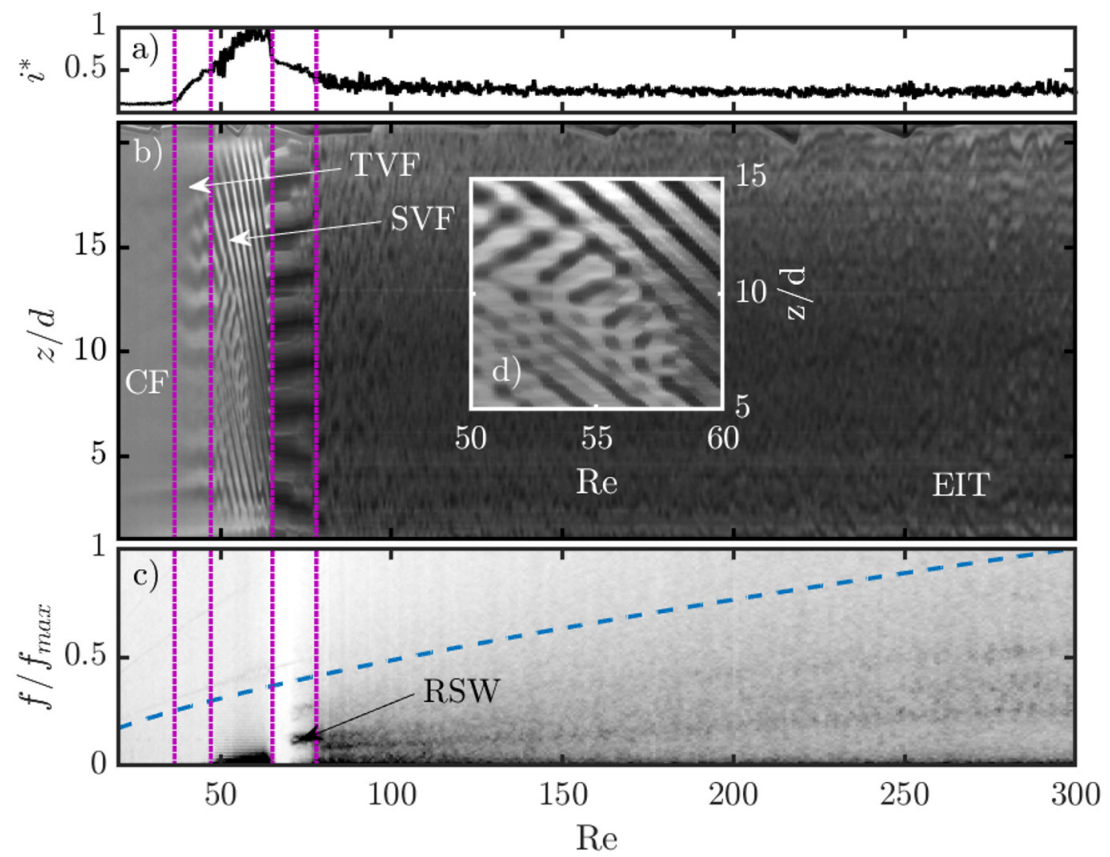

Figure 13: Intensity signal (a), flow map (b) and frequency map (c) for a highly elastic, highly shear-thinning fluid (XG2000-50-2 experiment, $\overline{\mathrm{El}}=74, \bar{n}_{e}=0.56$ ). The dashed line on sub-figure c denotes the inner cylinder rotation frequency. Vertical dotted lines denote the transitions between flow states. Note that the rotation frequency detected varies nonlinearly with Re, due to the shear-thinning behaviour. d) is a zoom of b) in the SVF state.

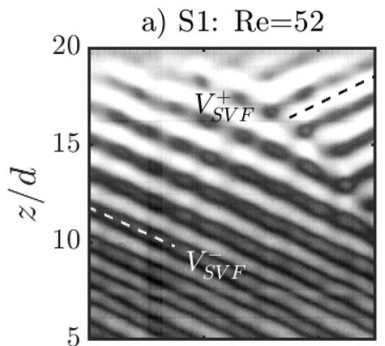

$\Delta t=50 \mathrm{~s}$ b) $\mathrm{S} 2: \mathrm{Re}=69$

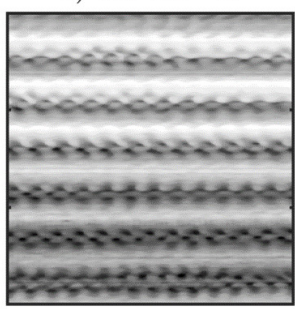

$\Delta t=10 \mathrm{~s}$ c) $\mathrm{S} 3: \mathrm{Re}=79$

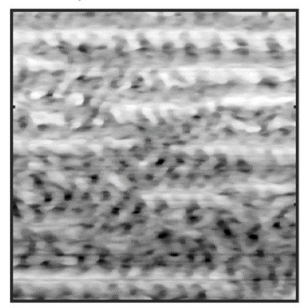

$\Delta t=10 \mathrm{~s}$ d) $\mathrm{S} 4: \mathrm{Re}=152$

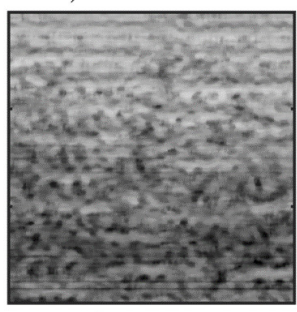

$\Delta t=10 \mathrm{~s}$

Figure 14: Steady state experiments in the SVF (S1), RSW (S2) and EIT (S3, S4) states, shown on $\Delta t$ intervals (horizontal axis) (S1 interval is larger in order to better describe the SVF state). Superscripts "-" and "+" in S1 denote upward and downward spiralling velocities, respectively.

thinning and viscoelastic PEO solutions, a local reduction in the amplitude of either of the two spirals led to spatial defects, the multiplication of which was a mechanism for the transition to EIT. A subregion of the flow map in figure $13 \mathrm{~b}$ ) in the (I)SVF state is shown in detail in figure $13 \mathrm{~d}$ ). SVF can be found to propagate either upwards or downwards. In highly shear-thinning XG solutions, it thus appears that one of the spirals can be completely suppressed, either on the full height of the cylinder (figures $13 \mathrm{~b}$ ), 13 d), 14 S1), or locally (Elçiçek \& Güzel 2020b). SVF or ISVF could thus be thought of as 

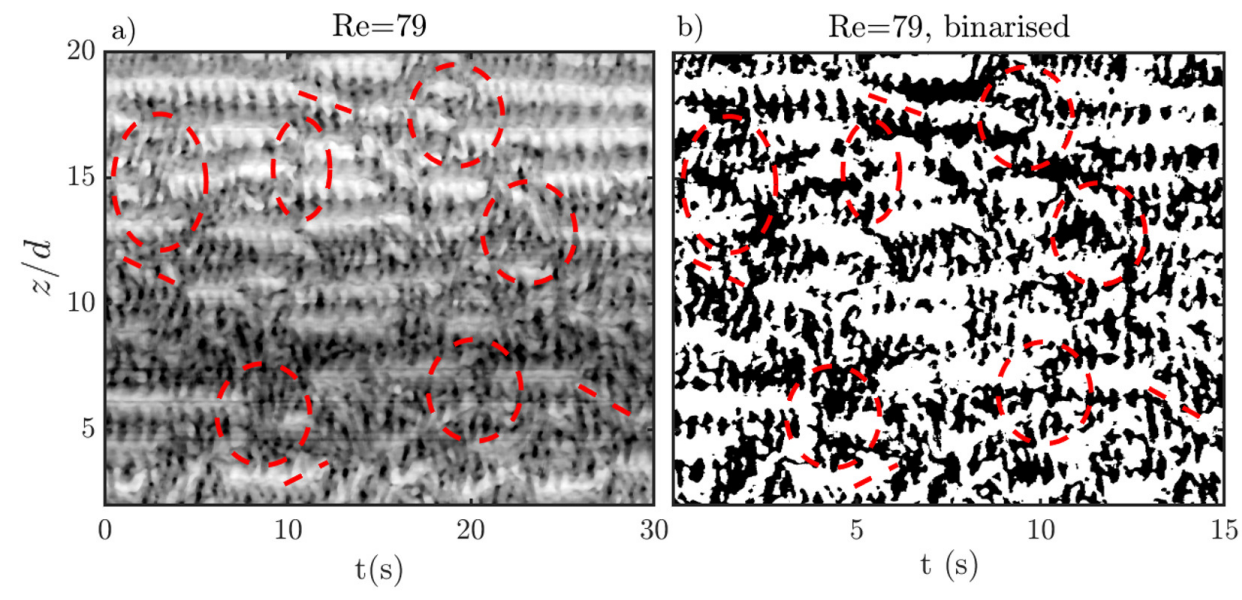

Figure 15: Steady state experiment (30 s) showing the transitional stage between RSW and EIT in highly elastic and shear-thinning XG2000-50 fluid, at Re =79. a) Reflected intensity, b) binarized intensity map. Some of the numerous merging and splitting events of Taylor-Vortices are highlighted by dashed circles, and the associated vortex drifting by dashed lines.

an alternative version of the RIB state, whereby shear-thinning combined with elasticity prevent spirals from co-existing everywhere in the gap.

Complementary steady state experiments have been performed and reported in table 3 and figure 14. This allows the spiral travelling speed to be computed for a constant Re $=52$ value: $V_{S V F}=d \times \alpha$, where $\alpha$ (expressed in $\mathrm{s}^{-1}$ ) is the slope of iso-intensity lines on a time-(normalized) space flow map (time versus $z / d$, figures $4 \mathrm{e}$ ), $14 \mathrm{a}$ )). It is found that $V_{S V F}^{-}=-0.84 \mathrm{~mm} . \mathrm{s}^{-1}$ in the downward spiralling section of the steady state experiment (figure 14, S1, $t<40 s, z / d<15$, lower dashed line). In the upper right corner of figure 14, S1 , an upward spiralling region is observed, with $V_{S V F}^{+}=0.90 \mathrm{~mm} . \mathrm{s}^{-1}$ (dark dashed line). The very close magnitude of these two velocities suggests that SVF and RIB spirals are related, but that SVF corresponds to a bistable state, where a wave of given velocity may travel in either direction, while the RIB state requires both waves to coexist.

The steady state experiment in the RSW state (at Re $=69$, sub-figure $14 \mathrm{~b}$ ), shows a pattern similar to that of the mild shear-thinning and elastic case XG200-50 in figure 11. With increasing Re, RSW state becomes increasingly disordered (figure 14 S3) and ultimately transitions to EIT (S4). In figure 15, the visualisation of figure $14 \mathrm{~S} 3$ ) is extended to a larger time span. Base Taylor vortices are crossed by multiple RSW waves, and the occurrence of several merge-split events and associated drifting of such base Taylor vortices are observed, leading to increased chaotic behaviours. Some merge-split and drifting features are indicated in figure 15 by dashed circles and lines respectively. This Merge-Split transition (MST) is a mechanism of transition to EIT in elastic, Boger fluids (Lacassagne et al. 2020), and it appears that it also applies here in the case of a highly elastic but also shear-thinning fluid.

Finally, from the steady state experiment above (figure 14), two-dimensional spacetime spectra can be computed as a means to probe the spatio-temporal dynamics of EIT, in this case in a shear-thinning fluid. In the RSW state, a spatial peak exists at $\lambda / d \sim 1$, due to the base TVF (figure 16 a). In the temporal dimension, several clear 

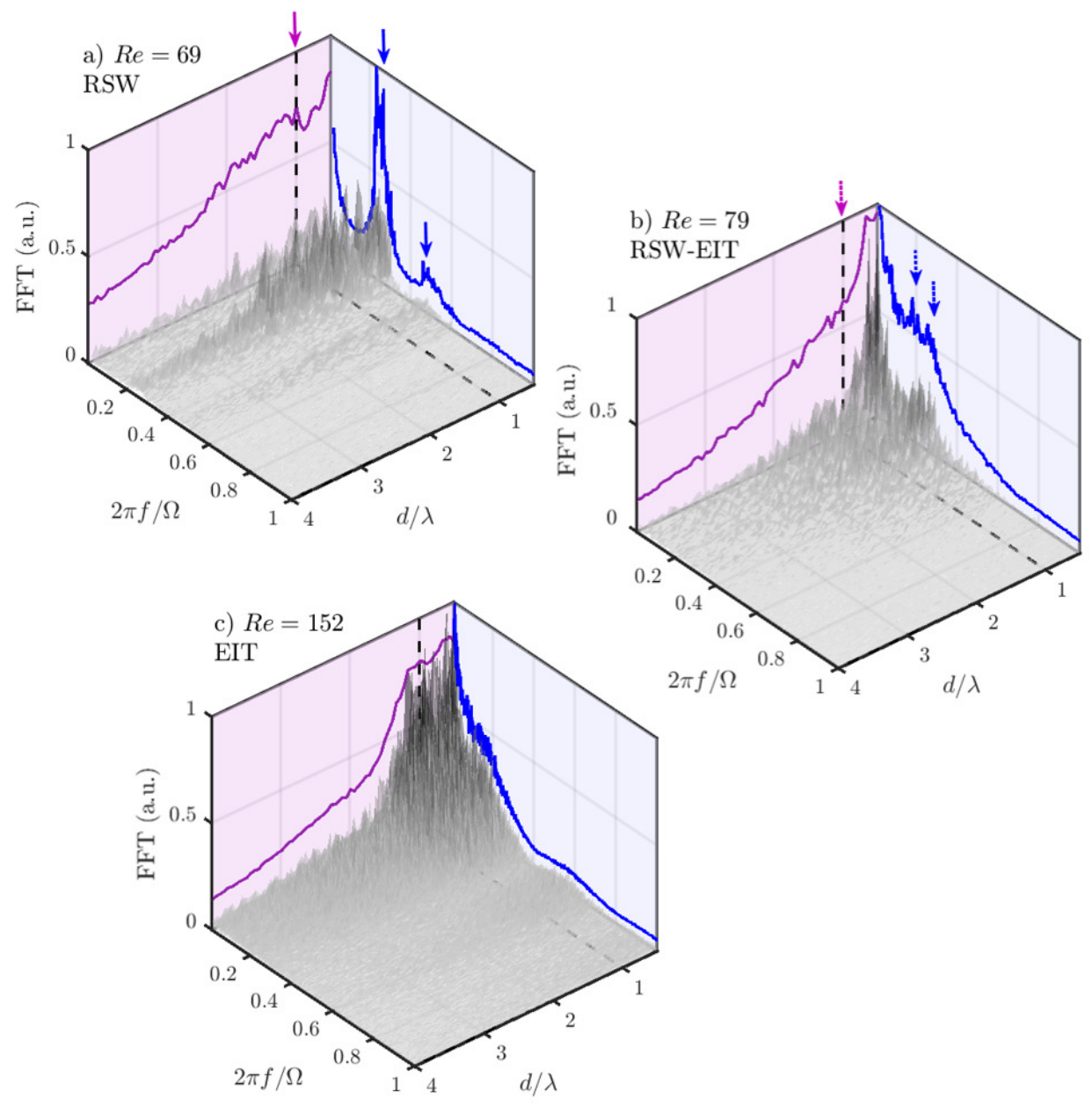

Figure 16: 2D FFT (time and space) of steady state experiments for the highly elastic, highly shear-thinning fluid (XG2000-50-2 experiment, $\overline{\mathrm{El}}=74, \bar{n}_{e}=0.56$ ) at $\operatorname{Re}=69$ (a), $\mathrm{Re}=79(\mathrm{~b})$ and $\mathrm{Re}=152$ (c) corresponding respectively to RSW, RSW transitioning to EIT, and EIT. Spectra are plotted in arbitrary units along a temporal frequency axis scaled by the inner cylinder rotation frequency $\Omega / 2 \pi$, and a spatial wavelength axis where the wavelength is scaled by the gap width $\mathrm{d}$. Space-averaged and time averaged 1D spectra are plotted in time-magnitude (blue) and space-magnitude (purple) planes respectively. Vertical arrows are guide to the eye showing peaks in both dimensions.

peaks are identified, the most energetic found at frequencies much lower than the rotation frequency of the inner cylinder (figures 7 c, 10 c, 11 h, (Lacassagne et al. 2020)).

In the transitional RSW-EIT state (figure $16 \mathrm{~b}$ ) several peaks can be seen in the temporal spectra, albeit broader. The spatial peak corresponding to TVF is reduced in relative amplitude, and the most dominant one is found for $\lambda / d<1$. The spatial structure of the flow is no longer dominated by the Taylor-Vortex wavelength, but by RSW waves of smaller wavelengths. Ultimately, EIT (figure $16 \mathrm{c}$ ) displays smoother, broadband spectra of multiple spatial and temporal scales, which correspond well to the 
common definition of turbulence (Fenstermacher et al. 1979; Dutcher \& Muller 2013; Liu \& Khomami 2013).

\section{Discussion}

In this section, the striking effect of shear-thinning mediation (attenuation and even suppression) of elastic flow patterns and EIT is discussed. First, the critical conditions for the onset of EIT in shear-thinning fluids are reported. Data from the present work and experiments from the literature are then compiled to derive an empirical criterion for EIT suppression in strongly shear-thinning fluids, and possible mechanisms for such behaviour are discussed.

\subsection{Effect of shear-thinning on the transition to EIT}

The transition to EIT, for some polymer solutions, is detected from the flow maps of ramp up experiments. Values of all parameters at the critical point are denoted using the superscript EIT and subscript $c$. Note that while some parameters are constant throughout the ramp up in Boger fluids (El, viscosity ratios etc.) their value depends on the shear-rate-variable viscosity in shear-thinning fluids.

In figure $17 \mathrm{a}$, the critical value $\operatorname{Re}_{c}^{E I T}$ is plotted as a function of $1-\bar{n}_{e}$ with colours representing the magnitude of $\overline{\mathrm{El}}$. At first, increasing the shear-thinning behaviour leads to a decrease in the critical Re for the onset of EIT, with a simultaneous increase of elasticity. Above a critical shear-thinning index of 0.82 is reached $\left(1-\bar{n}_{e}=0.18\right)$, shearthinning appears to delay the onset of EIT, despite the simultaneous significant increase in $\overline{\mathrm{El}}$ (figure $17 \mathrm{a}$ ).

A related non-monotonic trend can also be observed in figure $17 \mathrm{~b}$ ) where $\operatorname{Re}_{c}^{E I T}$ is this time plotted against $\overline{\mathrm{El}}$, with colour representing $1-\bar{n}_{e}$ values. For $\overline{\mathrm{El}}<1$, increasing $\overline{\mathrm{El}}$ together with a moderate decrease in the shear-thinning index $\left(\bar{n}_{e}\right.$ varying from 1 down to 0.82 ) leads to a decrease in $\operatorname{Re}_{c}^{E I T}$. Further increase of $\overline{\text { El }}$ above unity, together with a significant increase of the shear-thinning property, is associated with a delay of EIT.

The elasticity versus shear-thinning parameter space thus seems to indicate the existence of a critical point at $\bar{n}_{e}=0.82$ and $E l \sim 1$. For $0.82>\bar{n}_{e}>1$, shear-thinning is not sufficiently strong to disrupt the elastic destabilisation of the flow and the triggering of EIT. However for $\bar{n}_{e}<0.82$, even a large increase in elasticity is not sufficient to promote the onset of EIT, which is delayed by shear-thinning. In other words, for fluids with sufficiently strong shear-thinning rheology, the elastic instabilities are delayed by the shear-thinning behaviour. This trend can be seen by plotting the critical values of $\mathrm{El}$ at the onset of EIT as a function of the critical values of $1-n_{e}$ at this same onset in figure $17 \mathrm{c}$ ). It is found that while $E l<1$ is sufficient to trigger elastic instabilities for $0.82>n_{e}>1$ (region R1 in sub-figure c), much higher values of El (several orders of magnitude) are required for the onset of EIT if $n_{e}<0.82$ (region R2).

\subsection{Suppression of EIT}

Although EIT is found to be delayed due to shear-thinning (eg. figure 13) in some experiments, it is not evidenced for Re $<300$ in many other experiments reported here. Newtonian-like transition patterns (figure 8), or RSW transitioning to WTVF (figure 10) have been observed instead. In figure 18, data from the present study (circles for XG, square for PAAM) are plotted together with experiments from the literature in a shearthinning versus elasticity parameter space $\left(1-\bar{n}_{e}, \overline{\mathrm{El}}\right)$. Experiments for which EIT has been reported in the Reynolds range measured (indicated in table 4) are denoted using 

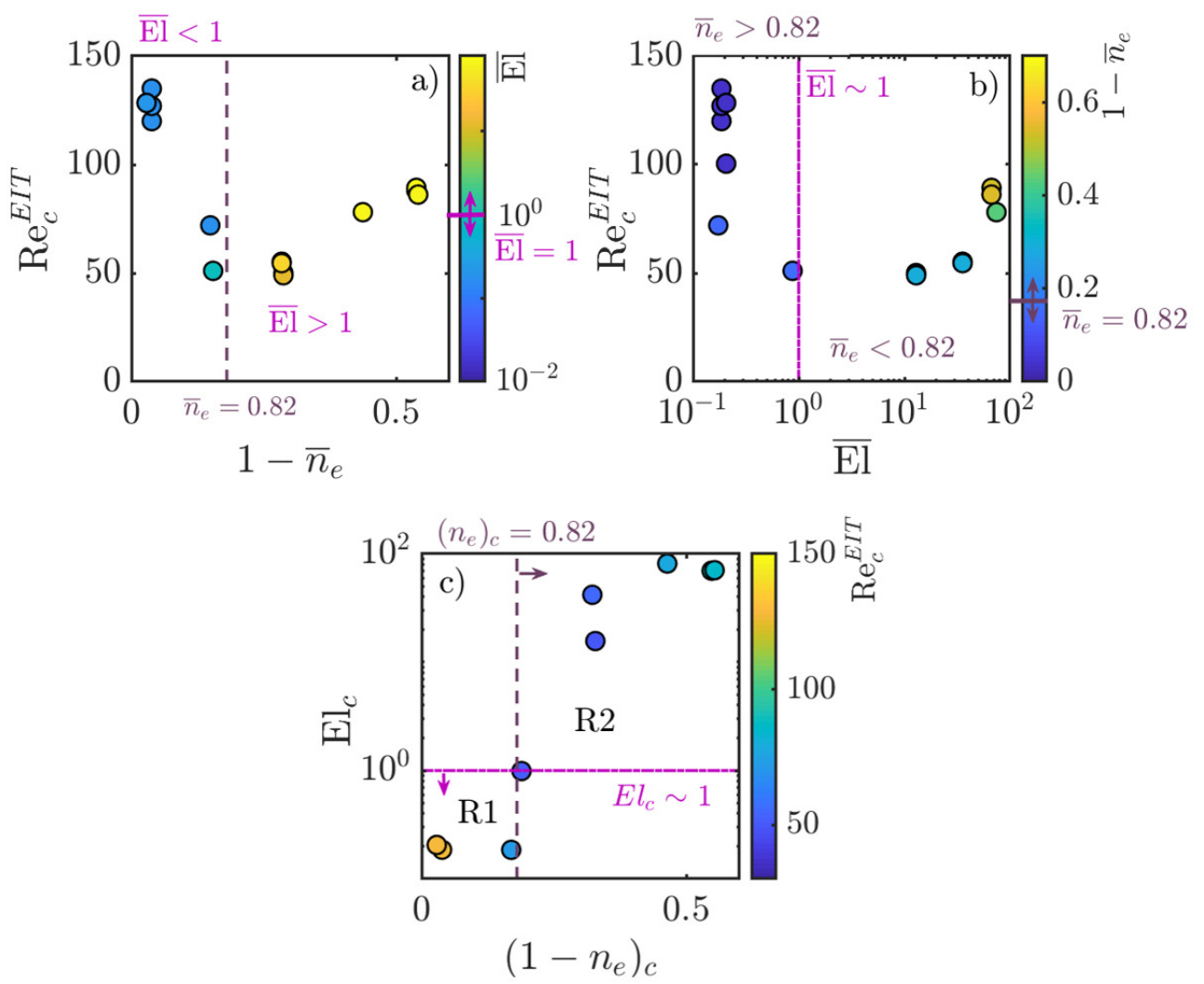

Figure 17: Critical Reynolds number value for the onset of EIT $\operatorname{Re}_{c}^{E I T}$ as a function of the averaged shear-thinning index (a) and elastic number (b). Marker colours on a and b indicate the values of $\overline{\mathrm{El}}$ and $1-\bar{n}_{e}$, respectively. Vertical lines are plotted at $n_{e}=0.82$ and $\overline{\mathrm{El}}=1$ on a and $\mathrm{b}$ respectively, and those threshold values are also reported on the colour-map of the conjugate figure (b and a respectively) as horizontal tick marks with arrows. Finally, sub-figure c) reports the critical values of $\mathrm{El}$ at the onset of $\mathrm{EIT}_{\mathrm{T}} \mathrm{El}_{c}$, as a function of the critical shear-thinning $1-n_{e}$ also at the onset. $\overline{\mathrm{El}}=1$ and $\bar{n}_{e}=0.82$ thresholds are noted as horizontal and vertical dashed lines, respectively.

white markers, while those for which EIT has not been observed (including the XG200-50 case with occasional RSW state, see figure 10) using black symbols. The flow transition patterns observed in these studies are also reported in table 4.

It should be noted that the data from the literature are not always fully characterised. For example in Groisman \& Steinberg (1997), it is mentioned that the "apparent solution viscosity was decreasing with increasing shear rate", but the degree of shear-thinning was not quantified. However their solvent viscosity was sufficiently high for the fluid to exhibit mild shear-thinning at most, and their results are well within the moderate El range. A study involving wormlike micellar solutions (WMS) is also reported (Mohammadigoushki \& Muller 2017), for which we estimate the shear-thinning index from the steady shear rheological characterisation reported by the authors (figure 1).

The $\left(1-\bar{n}_{e}=0, \overline{\mathrm{El}}\right)$ axis of figure 18 shows the existence of a critical value for $\overline{\mathrm{El}}$ beyond which EIT emerges for non-shear-thinning fluids. Below this curve, the 


\begin{tabular}{|c|c|c|c|c|c|c|c|c|}
\hline Reference & Additive & $A R$ & $\eta$ & $\frac{\mu_{p}}{\mu}$ & $\bar{n}_{e}$ & $\mathrm{El}$ & $\operatorname{Re}_{\max }$ & Pattern \\
\hline Present study & $\begin{array}{l}\text { XG, } \\
\text { PAAM }\end{array}$ & 21.6 & 0.770 & $\begin{array}{l}0.37- \\
1.0\end{array}$ & $\begin{array}{l}0.46- \\
1.0\end{array}$ & $\begin{array}{l}0.14- \\
74\end{array}$ & 300 & Various \\
\hline $\begin{array}{l}\text { Cagney et al. } \\
(2020)\end{array}$ & $\mathrm{XG}$ & 21.6 & 0.770 & $\begin{array}{l}0.13- \\
1.0\end{array}$ & $\begin{array}{l}0.46- \\
0.90\end{array}$ & $\begin{array}{l}0.053- \\
9.03\end{array}$ & 1000 & $\begin{array}{l}\text { CF- } \\
\text { TVF- } \\
\text { WTVF }\end{array}$ \\
\hline $\begin{array}{l}\text { Groisman \& Stein- } \\
\text { berg }(1996)\end{array}$ & PAAM & 54.0 & 0.708 & $\begin{array}{l}0.0083- \\
0.25\end{array}$ & N.A. & $\begin{array}{l}0.09- \\
0.34\end{array}$ & N.A. & $\begin{array}{l}\text { CF- } \\
\text { (TVF)- } \\
\text { (RSW)- } \\
\text { EIT }\end{array}$ \\
\hline $\begin{array}{l}\text { Crumeyrolle et al. } \\
(2002)\end{array}$ & PEO & 46.6 & 0.883 & $\begin{array}{l}0.16- \\
0.92\end{array}$ & $\begin{array}{l}0.90- \\
0.98\end{array}$ & $\begin{array}{l}0.0018- \\
0.050\end{array}$ & 200 & $\begin{array}{l}\text { CF- } \\
\text { TVF- } \\
\text { WTVF } \\
\text { or CF- } \\
\text { TVF- } \\
\text { RIB }\end{array}$ \\
\hline $\begin{array}{l}\text { Dutcher \& Muller } \\
(2011)\end{array}$ & PEO & 60.7 & 0.912 & 0.44 & 1.00 & $\begin{array}{l}0- \\
0.023\end{array}$ & $200-250$ & $\begin{array}{l}\text { CF- } \\
\text { TVF- } \\
\text { WTVF }\end{array}$ \\
\hline $\begin{array}{l}\text { Dutcher \& Muller } \\
(2013)\end{array}$ & PEO & 60.7 & 0.912 & 0.84 & 0.85 & $\begin{array}{l}0.1- \\
0.2\end{array}$ & $200-250$ & $\begin{array}{l}\text { CF- } \\
\text { TVF- } \\
\text { RSW- } \\
\text { EIT }\end{array}$ \\
\hline $\begin{array}{l}\text { Latrache et al. } \\
(2016)\end{array}$ & PEO & 46.6 & 0.883 & $\begin{array}{l}0.83- \\
0.96\end{array}$ & $\begin{array}{l}0.80- \\
0.92\end{array}$ & $\begin{array}{l}0.011- \\
0.14\end{array}$ & 150 & $\begin{array}{l}\text { CF- } \\
\text { TVF- } \\
\text { RIB- } \\
\text { EIT }\end{array}$ \\
\hline $\begin{array}{l}\text { Martínez-Arias \& } \\
\text { Peixinho }(2017)\end{array}$ & PEO & 30.0 & 0.909 & $\begin{array}{l}0.083- \\
0.63\end{array}$ & $\begin{array}{l}0.92- \\
1.00\end{array}$ & $\begin{array}{l}0.06- \\
1.09\end{array}$ & 200 & $\begin{array}{l}\text { CF- } \\
\text { TVF- } \\
\text { RSW- } \\
\text { EIT }\end{array}$ \\
\hline $\begin{array}{l}\text { Mohammadigoushki } \\
\text { \& Muller (2017) }\end{array}$ & WMS & 60.7 & 0.91 & $\sim 1.00$ & $\begin{array}{l}0.58- \\
0.82\end{array}$ & $\begin{array}{l}0.2- \\
3.8\end{array}$ & 100 & $\begin{array}{l}\mathrm{CF}- \\
(\mathrm{TVF} / \mathrm{SVF})- \\
\mathrm{EIT}\end{array}$ \\
\hline
\end{tabular}

Table 4: Flow transition patterns in shear-thinning and viscoelastic polymer or micellar solutions. N.A denotes quantities non-available or non-assessable from the available data. 


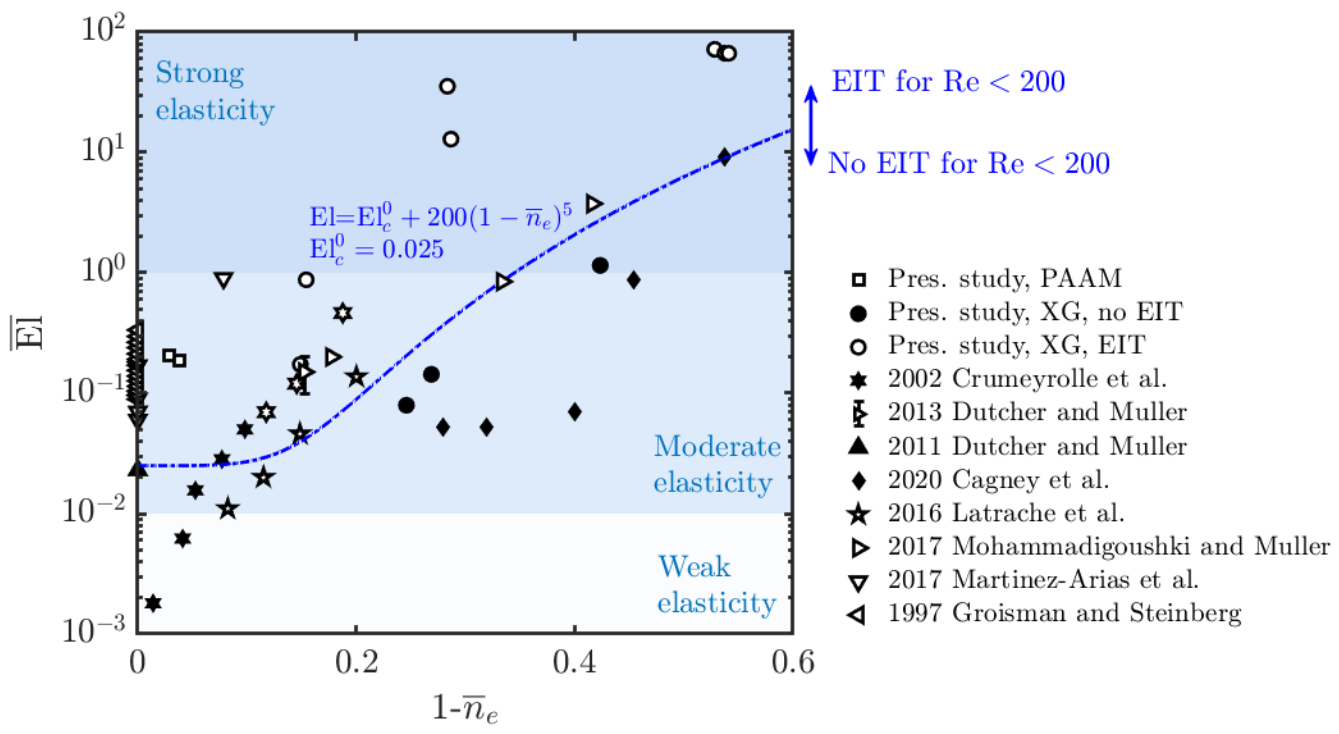

Figure 18: Shear-thinning versus elasticity parameter map in Taylor-Couette flows of polymeric solutions. Present study and literature experiments in the $\left(1-\bar{n}_{e}, \overline{\mathrm{El}}\right)$ parameter space. White symbols indicate reported EIT, and black ones absence of it, for $\operatorname{Re}<\operatorname{Re}_{\max }$ as reported in table 4 . The flow transition patterns observed in these studies are also reported in table 4 .

transitions are Newtonian-like due to the weak elasticity. This threshold value increases with increased shear-thinning (increasing $1-\bar{n}_{e}$ ), so that for highly shear-thinning fluids, no EIT may be observed, even in the high elasticity domain (figure 8). It may be attributed to the fact that EIT is completely suppressed by shear-thinning, or that it is simply delayed to $\mathrm{Re}_{c}$ values beyond the range studied. From comparison with the literature (figure 18, table 4), it appears that this phenomenon is common to a variety of polymers (including PEO (Crumeyrolle et al. 2002; Latrache et al. 2016), PAAM (Groisman \& Steinberg 1996) and XG (Cagney et al. 2020), present study) and even to some extent to WMS (e.g. Mohammadigoushki \& Muller (2017)). It becomes apparent that EIT needs both high El and significant Re to develop, but that shear-thinning may act to delay or even suppress elastic instabilities leading to EIT.

This can be expressed by an empirical criterion of the form

$$
\overline{\mathrm{El}}>\mathrm{El}_{c}^{0}+K \times\left(1-\bar{n}_{e}\right)^{\kappa}
$$

where $\mathrm{El}_{c}^{0}=0.025, K=200$ and $\kappa=5$ are empirical parameters adjusted visually in order for the empirical line of equation 4.1 to separate the two domains (empty and full marker clouds) both in strong and weak shear thinning regions in figure 18. The first one corresponds to the El threshold for Boger fluids, while the second and third ones describe the dependency of the El threshold on the shear-thinning index. Discrepancies in the data, especially for moderate shear-thinning $\left(1-\bar{n}_{e} \sim 0.1\right)$ and elasticity may be attributed to the different geometrical parameters of the study reported (aspect ratio and radius ratio). It is indeed known that the streamline curvature has a great impact on the onset of purely elastic instabilities (Pakdel \& McKinley 1996; Schaefer et al. 2018), and could similarly affect elasto-inertial instabilities. The empirical constants are thus likely to be 
related primarily to those geometrical parameters, and physical parameter of the fluid (temperature, molecular weight, shear-thinning viscosity, viscosity ratio etc) embedded into the $\overline{\mathrm{El}}$ and $\bar{n}_{e}$ estimators. On the other hand, figure 18, table 4 and equation 4.1 show that data collected from experimental setups different various geometrical parameters and using various polymer or micellar solutions all follow this empirical behaviour. This could possibly suggest, if not a universal behaviour, at least a weak influence of some of the geometrical parameters in the shear-thinning mediation process.

\subsection{What are the mechanisms of shear-thinning mediation?}

The mechanisms for mediation of elasto-inertial features by shear-thinning remain to be explained. Intuitively, one may first postulate that for strong shear-thinning, radial viscosity gradients exist within the gap due to the strong base azimuthal flow, leading to a low viscosity inner core, and a high viscosity outer region. Viscous dissipation would then happen over a narrow range and an effective gap, $\mathrm{d}^{r}<d$, would exist, with $r_{o}^{r}<r_{o}$, $r_{o}^{r}$ being the outer radius of the flowing region. This is comparable to the flow of shearbanding solutions (Perge et al. 2014).

The implications of this can be explored using simple scaling relations. Primarily, in the reduced gap, the apparent shear-rate for a given rotation speed increases: $\dot{\gamma}^{r}=\Omega r_{i} / d^{r}>$ $\dot{\gamma}$. As a direct consequence, the Weissenberg number is increased:

$$
\mathrm{Wi}^{r}=\dot{\gamma}^{r} t_{e}>\mathrm{Wi}
$$

The apparent viscosity in the inner core (subject to higher shear rates $\dot{\gamma}^{r}>\dot{\gamma}$ ) is expected to be lower than the apparent global viscosity in the full gap. Consequently, both the numerator and the denominator in the Re definition are reduced as a result of the gap reduction (equation 1.1). Simplifying the viscosity shear-rate dependency to a power law behaviour (introduced in section 1), one gets $\mu^{r} \sim\left(\dot{\gamma}^{r}\right)^{n-1}$, with typically $-0.6<n-1<$ 1. Since $\dot{\gamma}^{r} \sim 1 / d^{r}$, it follows that $\mu^{r} \sim\left(d^{r}\right)^{1-n}$, and

$$
\operatorname{Re}^{r} \sim \frac{\gamma^{r}\left(d^{r}\right)^{2}}{\mu^{r}} \sim \frac{\left(d^{r}\right)^{-1}\left(d^{r}\right)^{2}}{\left(\left(d^{r}\right)^{-1}\right)^{n-1}} \sim\left(d^{r}\right)^{n},
$$

and finally,

$$
\mathrm{El} \sim\left(d^{r}\right)^{-n-1} .
$$

Since $0<n<1$, shear-thinning will cause a decrease in $\mathrm{d}^{r}$ which corresponds to a weak non-linear decrease in Re, but more importantly an increase in El. Thus, shearthinning is not likely to be the case of reduced elastic instability, since it is conversely expected to promote elastic properties (i.e. increase $\mathrm{Wi}, \mathrm{El}$ ).

While this analysis has been based on a purely azimuthal $\mathrm{CF}$, the radial viscosity gradient due to the main shear in the gap persists even for higher order flows. For example in Cagney \& Balabani (2019b); Topayev et al. (2019), it is shown that Taylor vortices are squeezed and deform against the inner cylinder, in the lower viscosity inner region (Topayev et al. 2019). The previous scaling arguments on enhanced elasticity parameters are thus likely to hold in more complex flows as well due to the base mean shear. On the other hand, secondary flows such as Taylor vortices are also affected by shear-thinning (Cagney \& Balabani 2019b; Topayev et al. 2019), which in turn leads to axial viscosity gradients. Such axial viscosity gradients could well be involved in the damping effect of shear-thinning on transverse elastic waves. Results from section 3.5 also suggest that shear-thinning damping of elastic waves must involve an interplay between inertia and shear-thinning.

The effects of shear-thinning could thus be interpreted through a concept of preferential 
flow paths, outside of which elastic waves may not be able to travel. Axial elastic perturbations would thus be confronted with axial viscosity gradients which may act as dampers. Moreover, the strength of the extensional deformations induced by such perturbations would be reduced compared to the dominant radial shear rate, which is also in turn enhanced by the radial viscosity distribution. An analogy to this mechanism can be found in the recent work by Walkama et al. (2020), who performed an experimental study of the onset of elastic instabilities (at vanishing Re) in a microfluidic flow of PEObased Boger fluids past an array of cylinders. Spatial disorder was found to delay or even suppress the onset of elastic instability. This was explained by the establishment, of preferential flow paths governed by the geometry, where shear deformation of the polymer is promoted over extensional deformation, having thus a stabilizing effects on the polymer chains. Interestingly their results were also compared with a similar experiment using a shear-thinning 3000 ppm xanthan gum solution (which may have featured significant elasticity, however not quantified). No elastic instabilities were observed on a similar range of $\mathrm{Wi}$ values despite the zero spatial disorder imposed, suggesting that shearthinning may act to suppress elastic disorder with mechanisms comparable to those of spatial disorder.

It should be mentioned that instabilities and flow transition in viscoelastic fluids are often found to be hysteretic and strongly subcritical (Martínez-Arias \& Peixinho 2017; Groisman \& Steinberg 1996). If one were to characterise the nature of the bifurcations, ramp-down experiments (decreasing inner cylinder rotation speed) would be needed. From the results of Martínez-Arias \& Peixinho (2017), there is no reason to expect shear-thinning to completely suppress hysteretic behaviours. It would yet be of great interest to see how the conclusions of the present study apply to flow states encountered specifically during ramp-down protocol only, such as diwhirls described in Groisman \& Steinberg (1996); Lange \& Eckhardt (2001); Martínez-Arias \& Peixinho (2017).

\section{Summary and conclusions}

In this work, Taylor-Couette flow of polymer solutions of various degrees of elasticity and shear-thinning were studied using flow-visualisation. Combined shear-thinning and elasticity were found to globally de-stabilize the azimuthal Couette flow and favour the onset of unsteady flow states with increased complexity (WTVF, SVF), sometimes transitioning to chaotic and seemingly turbulent (RSW, EIT) even at relatively low Re.

Shear-thinning mediates the transition patterns of moderately to highly elastic polymer solutions. In the absence of shear-thinning (Boger fluids), the flow transitioned to EIT even at moderate elasticity levels. In contrast, in highly shear-thinning cases, moderate to even high elasticity fluids may undergo Newtonian-like transition patterns, for which no elasto-inertial instabilities occurred. Features of the flow states, such as the TVF wavelength or the wavy frequency were also modified by the fluid's rheology. When both shear-thinning and elasticity were moderate, elasto-inertial flow states (RSW, EIT) were reported in some cases. It was found that their nature was modified by shear-thinning. An increase in inertia tended to suppress chaos in the flow. Finally, in the most highly elastic fluids that also had significant shear-thinning, the flow ultimately did transition to EIT in a complex fashion. The Merge-Split Transition (MST) mechanism reported in Lacassagne et al. (2020) for Boger fluids was also observed. However, the transition occurred at elastic number values much higher than those required in the absence of shear-thinning.

The global effect of shear-thinning was, thus, to delay or even suppress elasto-inertial flow states, delaying their onset, occasionally damping chaotic features of the flow 
when inertia was increased, or even completely suppressing EIT. For this last effect, an empirical criterion for the existence of EIT was derived, also using results from the literature on various polymer solutions. Experiments with additional polymers, WMS, with a fully characterized rheology and in different geometrical configurations would be of great interest to verify the universality of this criterion. The physical origin of the shearthinning mediation of elastic instabilities remains to be explained. It is not predicted by scaling arguments but could be the consequence of axial viscosity gradients caused by shear-thinning and secondary flows.

From a physical standpoint, both shear-thinning and viscoelastic properties originate from the same fluid component: polymer chains. Disentangling the two effects is extremely relevant from an empirical and applied point of view. It has, for example, recently been applied in order to explain flow phenomena in micro-fluidic devices (Casanellas et al. 2016; Haward et al. 2020). Casanellas et al. (2016) showed that in the absence of inertia, the Pakdel-McKinley criterion commonly used to describe the onset of purely elastic instabilities does not systematically capture the effects of shear-thinning. By properly characterising the fluid rheology, it may be possible to predict the existence, absence, or damping of elasto-inertial regimes in a Taylor-Couette flow without further knowledge or modelling of the state of polymer chains themselves. It is also evident that CF, TVF, WTVF or EIT may be associated with different degrees of mixing performances, related to flow unsteadiness and chaotic behaviour. Knowledge of very simple rheological fluid parameters could thus allow to foresee, for a given amount of inertia, the performance of a Taylor-Couette mixer in a shear-thinning and viscoelastic fluid. Tuning of such rheological parameters by controlling either the solvent viscosity or the polymer nature may in turn allow the mixing performances to be controlled and optimised.

\section{Acknowledgements}

Financial support for this work from the Engineering and Physical Sciences Research Council (EPSRC) Manufacturing the Future programme (No.EP/N024915/1) is gratefully acknowledged.

\section{Declaration of interests}

The authors report no conflict of interest.

\section{REFERENCES}

Abcha, N., Kelai, F., Latrache, N., Crumeyrolle, O. \& Mutabazi, I. 2018 Radial Propagation of the Instability Modes Observed in a Viscoelastic Couette-Taylor Flow. In Nonlinear Waves and Pattern Dynamics (ed. N. Abcha, E. Pelinovsky \& I. Mutabazi), pp. 181-196. Cham: Springer International Publishing.

Akonur, A. \& Lueptow, R. M. 2003 Three-dimensional velocity field for wavy Taylor-Couette flow. Physics of Fluids 15 (4), 947-960.

Alibenyahia, B., Lemaitre, C., Nouar, C. \& Ait-Messaoudene, N. 2012 Revisiting the stability of circular Couette flow of shear-thinning fluids. Journal of Non-Newtonian Fluid Mechanics 183-184, 37-51.

Andereck, C.D., Liu, S.S. \& Swinney, H.L. 1986 Flow regimes in a circular Couette system with independently rotating cylinders. Journal of Fluid Mechanics 164, 155-183.

Ashrafi, N. \& Khayat, R. E. 2000 Shear-thinning-induced chaos in Taylor-Couette flow. Physical Review E 61 (2), 1455-1467.

Avgousti, M. \& Beris, A. N. 1993 Non-axisymmetric modes in viscoelastic taylor-couette flow. Journal of Non-Newtonian Fluid Mechanics 50 (2), 225-251. 
Bahrani, S. A., Nouar, C., Neveu, A. \& Becker, S. 2015 Transition to chaotic TaylorCouette flow in shear-thinning fluids. p. 11. Lyon.

Barlow, H. J., Hemingway, E. J., Clarke, A. \& Fielding, S. M. 2019 Linear instability of shear thinning pressure driven channel flow. Journal of Non-Newtonian Fluid Mechanics 270, 66-78.

Baumert, B. M. \& Muller, S. J. 1997 Flow regimes in model viscoelastic fluids in a circular couette system with independently rotating cylinders. Physics of Fluids 9 (3), 566-586.

Baumert, B. M. \& Muller, S. J. 1999 Axisymmetric and non-axisymmetric elastic and inertio-elastic instabilities in Taylor-Couette flow. Journal of Non-Newtonian Fluid Mechanics 83 (1), 33-69.

Bodiguel, H., Beaumont, J., Machado, A., Martinie, L., Kellay, H. \& Colin, A. 2015 Flow Enhancement due to Elastic Turbulence in Channel Flows of Shear Thinning Fluids. Physical Review Letters 114 (2), 028302.

Boger, D. V. 1977 A highly elastic constant-viscosity fluid. Journal of Non-Newtonian Fluid Mechanics (3), 87-89.

Cagney, N. \& Balabani, S. 2019a Influence of Shear-Thinning Rheology on the Mixing Dynamics in Taylor-Couette Flow. Chemical Engineering 83 Technology 42 (8), 16801690.

Cagney, N. \& Balabani, S. $2019 b$ Taylor-Couette flow of shear-thinning fluids. Physics of Fluids 31 (5), 053102.

Cagney, Neil, Lacassagne, Tom \& Balabani, Stavroula 2020 Taylor-Couette flow of polymer solutions with shear-thinning and viscoelastic rheology. Journal of Fluid Mechanics 905.

Casanellas, L., Alves, M. A., Poole, R. J., Lerouge, S. \& Lindner, A. 2016 The stabilizing effect of shear thinning on the onset of purely elastic instabilities in serpentine microflows. Soft Matter 12 (29), 6167-6175.

Caton, F. 2006 Linear stability of circular Couette flow of inelastic viscoplastic fluids. Journal of Non-Newtonian Fluid Mechanics 134 (1), 148-154.

Chhabra, R. P. \& Richardson, J. F. 1999 Non-Newtonian Flow in the Process Industries: Fundamentals and Engineering Applications. Butterworth-Heinemann.

Cole, J. A. 1976 Taylor-vortex instability and annulus-length effects. Journal of Fluid Mechanics 75 (1), 1-15.

Coles, Donald 1965 Transition in circular Couette flow. Journal of Fluid Mechanics 21 (3), 385-425.

Coronado-Matutti, O., Souza Mendes, P. R. \& Carvalho, M. S. 2004 Instability of Inelastic Shear-Thinning Liquids in a Couette Flow Between Concentric Cylinders. Journal of Fluids Engineering 126 (3), 385-390.

Coughlin, K. T. \& Marcus, P. S. $1992 a$ Modulated waves in Taylor-Couette flow Part 1. Analysis. Journal of Fluid Mechanics 234, 1-18.

Coughlin, K. T. \& Marcus, P. S. $1992 b$ Modulated waves in Taylor-Couette flow Part 2. Numerical simulation. Journal of Fluid Mechanics 234, 19-46.

Crumeyrolle, O, Latrache, N, Mutabazi, I \& Ezersky, A B 2005 Instabilities with shearthinning polymer solutions in the Couette-Taylor system. Journal of Physics: Conference Series 14, 78-93.

Crumeyrolle, O., Mutabazi, I. \& Grisel, M. 2002 Experimental study of inertioelastic Couette-Taylor instability modes in dilute and semidilute polymer solutions. Physics of Fluids 14 (5), 1681-1688.

Divoux, T., Fardin, M. A., Manneville, S. \& Lerouge, S. 2016 Shear Banding of Complex Fluids. Annual Review of Fluid Mechanics 48 (1), 81-103.

Dutcher, C. S. \& Muller, S. J. 2009 Spatio-temporal mode dynamics and higher order transitions in high aspect ratio Newtonian Taylor-Couette flows. Journal of Fluid Mechanics 641, 85-113.

Dutcher, C. S. \& Muller, S. J. 2011 Effects of weak elasticity on the stability of high Reynolds number co- and counter-rotating Taylor-Couette flows. Journal of Rheology 55 (6), 1271-1295.

Dutcher, C. S. \& Muller, S. J. 2013 Effects of moderate elasticity on the stability of coand counter-rotating Taylor-Couette flows. Journal of Rheology 57 (3), 791-812. 
ElÇıÇEK, H. \& GüZEL, B. $2020 a$ Effect of shear-thinning behavior on flow regimes in Taylor-Couette flows. Journal of Non-Newtonian Fluid Mechanics 279, 104277.

ElÇIÇEK, H. \& GüZEL, B. $2020 b$ On non-axisymmetric flow structures of graphene suspensions in Taylor-Couette reactors. International Journal of Environmental Science and Technology .

Escudier, M. P., Gouldson, I. W. \& Jones, D. M. 1995 Taylor vortices in Newtonian and shear-thinning liquids. Proceedings of the Royal Society of London. Series A: Mathematical and Physical Sciences 449 (1935), 155-176.

Fardin, M. A., J. Ober, T., Grenard, V., Divoux, T., Manneville, S., H. McKinley, G. \& Lerouge, S. 2012 Interplay between elastic instabilities and shear-banding: three categories of Taylor-Couette flows and beyond. Soft Matter 8 (39), 10072-10089.

Fardin, M. A., Lopez, D., Croso, J., Grégoire, G., Cardoso, O., McKinley, G. H. \& Lerouge, S. 2010 Elastic Turbulence in Shear Banding Wormlike Micelles. Physical Review Letters 104 (17), 178303.

Fardin, M. A., Perge, C. \& Taberlet, N. 2014 "The hydrogen atom of fluid dynamics" introduction to the Taylor-Couette flow for soft matter scientists. Soft Matter 10 (20), 3523-3535.

Fenstermacher, P. R., Swinney, Harry L. \& Gollub, J. P. 1979 Dynamical instabilities and the transition to chaotic Taylor vortex flow. Journal of Fluid Mechanics 94 (1), $103-128$.

Gillissen, J.J.J. 2019 Two-dimensionnal decaying elastoinertial turbulence. Physical Review Letters 123 (14), 144502.

Groisman, A. \& Steinberg, V. 1996 Couette-Taylor Flow in a Dilute Polymer Solution. Physical Review Letters $\mathbf{7 7}$ (8), 1480-1483.

Groisman, A. \& Steinberg, V. 1997 Solitary Vortex Pairs in Viscoelastic Couette Flow. Physical Review Letters 78 (8), 1460-1463.

Groisman, A. \& Steinberg, V. 2000 Elastic turbulence in a polymer solution flow. Nature 405 (6782), 53.

Groisman, A. \& Steinberg, V. 2004 Elastic turbulence in curvilinear flows of polymer solutions. New Journal of Physics 6, 29-29.

Grossmann, S., Lohse, D. \& Sun, C. 2016 High-Reynolds Number Taylor-Couette Turbulence. Annual Review of Fluid Mechanics 48 (1), 53-80.

Gul, M., Elsinga, G. E. \& Westerweel, J. 2018 Experimental investigation of torque hysteresis behaviour of Taylor-Couette Flow. Journal of Fluid Mechanics 836, 635-648.

Haward, S. J., Hopkins, C. C. \& Shen, A. Q. 2020 Asymmetric flow of polymer solutions around microfluidic cylinders: Interaction between shear-thinning and viscoelasticity. Journal of Non-Newtonian Fluid Mechanics p. 104250.

Hopkins, Cameron C., Haward, Simon J. \& Shen, Amy Q. 2020 Purely Elastic Fluid-Structure Interactions in Microfluidics: Implications for Mucociliary Flows. Small 16 (9), 1903872.

James, D. F. 2009 Boger Fluids. Annual Review of Fluid Mechanics 41 (1), 129-142.

Lacassagne, T., Cagney, N., Gillissen, J. J. J. \& Balabani, S. 2020 Vortex merging and splitting: A route to elastoinertial turbulence in Taylor-Couette flow. Physical Review Fluids 5 (11), 113303.

Lange, M. \& Eckhardt, B. 2001 Vortex pairs in viscoelastic Couette-Taylor flow. Physical Review E 64 (2), 027301.

Larson, R.G. \& Desai, P. S. 2015 Modeling the Rheology of Polymer Melts and Solutions. Annual Review of Fluid Mechanics 47 (1), 47-65.

Larson, R. G. 2000 Turbulence without inertia. Nature 405 (6782), 27-28.

Larson, R. G., Muller, S. J. \& Shaqfeh, E. S. G. 1994 The effect of fluid rheology on the elastic Taylor-Couette instability. Journal of Non-Newtonian Fluid Mechanics $\mathbf{5 1}$ (2), $195-225$.

Larson, R. G., Shaqfeh, E. S. G. \& Muller, S. J. 1990 A purely elastic instability in Taylor-Couette flow. Journal of Fluid Mechanics 218, 573-600.

Latrache, N., Abcha, N., Crumeyrolle, O. \& Mutabazi, I. 2016 Defect-mediated turbulence in ribbons of viscoelastic Taylor-Couette flow. Physical Review E 93 (4), 043126 . 
Latrache, N., Crumeyrolle, O. \& Mutabazi, I. 2012 Transition to turbulence in a flow of a shear-thinning viscoelastic solution in a Taylor-Couette cell. Physical Review E 86 (5), 056305 .

Liu, N. \& Khomami, B. 2013 Elastically induced turbulence in Taylor-Couette flow: direct numerical simulation and mechanistic insight. Journal of Fluid Mechanics 737, R4.

Lockett, T. J., Richardson, S. M. \& Worraker, W. J. 1992 The stability of inelastic non-Newtonian fluids in Couette flow between concentric cylinders: a finite-element study. Journal of Non-Newtonian Fluid Mechanics 43 (2), 165-177.

Martínez-Arias, B. \& Peixinho, J. 2017 Torque in Taylor-Couette flow of viscoelastic polymer solutions. Journal of Non-Newtonian Fluid Mechanics 247, 221-228.

Masuda, H., Horie, T., Hubacz, R., Ohta, M. \& Ohmura, N. 2017 Prediction of onset of Taylor-Couette instability for shear-thinning fluids. Rheologica Acta 56 (2), 73-84.

Mohammadigoushisi, H. \& Muller, S. J. 2017 Inertio-elastic instability in Taylor-Couette flow of a model wormlike micellar system. Journal of Rheology 61 (4), 683.

Pakdel, P. \& McKinley, G. H. 1996 Elastic Instability and Curved Streamlines. Physical Review Letters 77 (12), 2459-2462.

Perge, C., Fardin, M. A. \& Manneville, S. 2014 Inertio-elastic instability of non shearbanding wormlike micelles. Soft Matter 10 (10), 1450-1454.

RAmesh, P. \& Alam, M. 2020 Interpenetrating spiral vortices and other coexisting states in suspension Taylor-Couette flow. Physical Review Fluids 5 (4), 042301.

Ramesh, P., BharadwaJ, S. \& Alam, M. 2019 Suspension Taylor-Couette flow: co-existence of stationary and travelling waves, and the characteristics of Taylor vortices and spirals. Journal of Fluid Mechanics 870, 901-940.

Schaefer, C., Morozov, A. \& Wagner, C. 2018 Geometric scaling of elastic instabilities in the Taylor-Couette geometry: A theoretical, experimental and numerical study. arXiv:1806.00328 [cond-mat, physics:physics] .

Sinevic, V., Kuboi, R. \& Nienow, A. W. 1986 Power numbers, Taylor numbers and Taylor vortices in viscous newtonian and non-newtonian fluids. Chemical Engineering Science 41 (11), 2915-2923.

Steinberg, V. 2019 Scaling Relations in Elastic Turbulence. Physical Review Letters 123 (23), 234501.

Steinberg, V. \& Groisman, A. 1998 Elastic versus inertial instability in Couette-Taylor flow of a polymer solution: Review. Philosophical Magazine B $\mathbf{7 8}$ (2), 253-263.

TAYlOR, G. I. 1923 Stability of a viscous liquid contained between two rotating cylinders. Philosophical Transactions of the Royal Society of London. Series A, Containing Papers of a Mathematical or Physical Character 223 (605-615), 289-343.

Thomas, D. G., Sureshiumar, R. \& Khomami, B. 2006 Pattern Formation in Taylor-Couette Flow of Dilute Polymer Solutions: Dynamical Simulations and Mechanism. Physical Review Letters 97 (5), 054501.

Topayev, S., Nouar, C., Bernardin, D., Neveu, A. \& Bahrani, S. A. 2019 Taylor-vortex flow in shear-thinning fluids. Physical Review E 100 (2), 023117.

Varshney, A. \& Steinberg, V. 2019 Elastic Alfven waves in elastic turbulence. Nature Communications 10 (1), 1-7.

VOlK, A. \& KäHLER, C. J. 2018 Density model for aqueous glycerol solutions. Experiments in Fluids 59 (5).

Walkama, D. M., Waisbord, N. \& Guasto, J. S. 2020 Disorder Suppresses Chaos in Viscoelastic Flows. Physical Review Letters 124 (16), 164501.

White, J. M. \& Muller, S. J. 2002a Experimental studies on the stability of Newtonian Taylor-Couette flow in the presence of viscous heating. Journal of Fluid Mechanics 462, 133-159.

White, J. M. \& Muller, S. J. $2002 b$ The role of thermal sensitivity of fluid properties, centrifugal destabilization, and nonlinear disturbances on the viscous heating instability in Newtonian Taylor-Couette flow. Physics of Fluids 14 (11), 3880-3890.

Zirnsak, M. A., Boger, D. V. \& Tirtanatmadja, V. 1999 Steady shear and dynamic rheological properties of xanthan gum solutions in viscous solvents. Journal of Rheology 43 (3), 627-650.

Öztekin, A., Brown, R. A. \& McKinley, G. H. 1994 Quantitative prediction of the 
a)

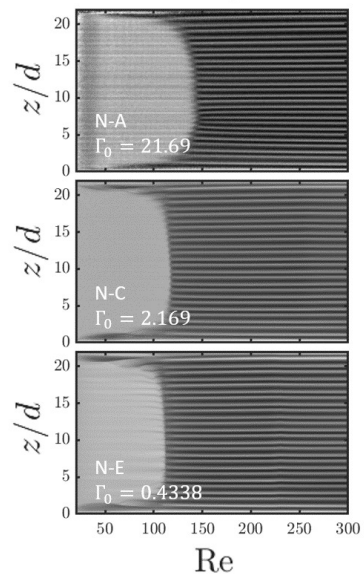

b)

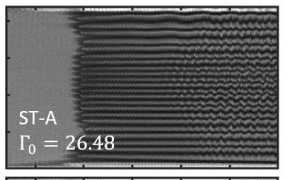

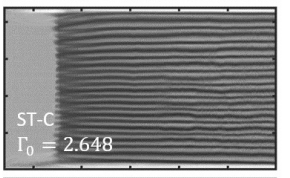

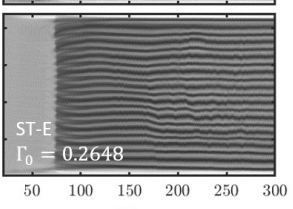

Re

c)
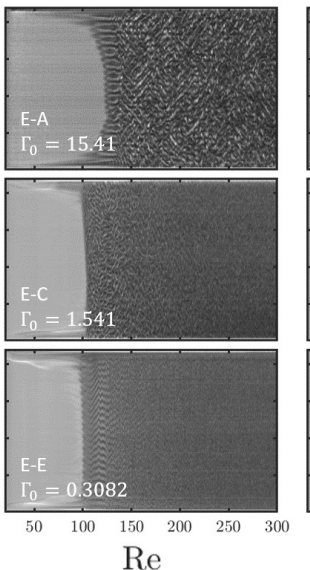

d)
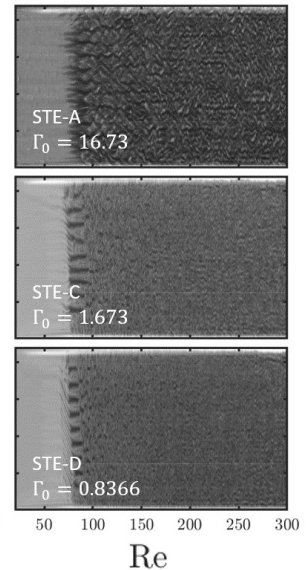

Figure 19: Flow maps of fastest (top row) to lowest (bottom row) raamp up experiments for a Newtonian fluid (N, first column, a), Shear-thinning fluid with a Newtonian-like transition (ST, second column, b), purely elastic Boger fluid (E, third column, c), and shear-thinning fluid with strong elasticity (STE, fourth column, d). The fluid type (N, $\mathrm{ST}, \mathrm{E}, \mathrm{STE}$ ), run index (A to E) and maximum NDAR $\Gamma_{0}$ are reported in each sub-figure.

viscoelastic instability in cone-and-plate flow of a Boger fluid using a multi-mode Giesekus model. Journal of Non-Newtonian Fluid Mechanics 54, 351-377.

\section{Appendix A. Effect of non-dimensional acceleration rate}

In this appendix, dedicated ramp up experiments are performed. For each fluid, several tests are performed, in which $\mathrm{Re}_{\max }$ is kept constant, and $\mathrm{d} \Omega / \mathrm{d} t$ and experiment duration are varied over several decades to induce variations of $\Gamma_{0}$.

Five fluids corresponding to four different typical behaviours are studied: a Newtonian case (N72, noted N), a shear-thinning dominated case case, with a Newtonian-like transition sequence (XG1000-25, noted ST), a purely elastic, Boger fluid (P500-72, noted E), and a fluid with shear-thinning but dominant elasticity (XG2000-72, noted STE). Experimental conditions for all tests are detailed in table 5 .

The frequency map tool can not be used in the following analysis. Indeed, when varying $\mathrm{d} \Omega / \mathrm{d} t$, the recording frequency has to be adapted in order to achieve similar Re-resolution flow maps regardless of the acceleration. In other words, $f_{s} / f_{\text {max }}$ has to be kept constant (typically above 3 ). Yet, the $N_{c}$ segment must be long enough to capture low frequency events, even at high frame rates. Capturing a similar wave frequency $f_{w}$, with the same $f_{s} / f_{\max }$ ratio, would require to increase $N_{c}$ proportionally to $f_{s}$ (in order of magnitude). The sampling time and Re variations corresponding to $N_{c}$ may then become too large to assume that Re stays constant within the sampled sequence. Frequency maps can thus not be used systematically to describe fast ramp up experiments, and we are here limited to the qualitative study of flow states and to the quantification of transitions through flow maps. This is however a physical process more due to the acceleration rate itself rather than an experimental limitation: ramping up faster than a typical wave time-scale does not allow a wave or perturbation with this time-scale to develop in a steady manner, and thus to be captured in a frequency map. 


\begin{tabular}{llllllll} 
Sample & Test & $f_{s}$ & $1 / \Delta R e$ & $\Omega_{\max }$ & $\mathrm{d} \Omega / \mathrm{d} t$ & $\Gamma_{0}$ & Expected behaviour \\
& & $\mathrm{Hz}$ & & $\mathrm{s}^{-1}$ & $\mathrm{~s}^{-2}$ & & \\
\hline N72 & $\mathrm{A}$ & 3000 & 179 & 66.96 & 3.732 & 21.69 & $\mathrm{~N}$ \\
$\mathrm{~N} 72$ & $\mathrm{~B}$ & 1500 & 179 & 66.96 & 1.866 & 10.85 & $\mathrm{~N}$ \\
$\mathrm{~N} 72$ & $\mathrm{C}$ & 300 & 179 & 66.96 & 0.3732 & 2.169 & $\mathrm{~N}$ \\
$\mathrm{~N} 72$ & $\mathrm{D}$ & 150 & 179 & 66.96 & 0.1866 & 1.085 & $\mathrm{~N}$ \\
N72 & $\mathrm{E}^{*}$ & 75 & 224 & 66.96 & 0.07463 & 0.4338 & $\mathrm{~N}$ \\
$\mathrm{~N} 72$ & $\mathrm{~F}^{*}$ & 30 & 202 & 66.96 & 0.03317 & 0.1928 & $\mathrm{~N}$ \\
XG1000-25 & $\mathrm{A}$ & 3000 & 200 & 46.84 & 2.229 & 26.48 & $\mathrm{ST}$ \\
XG1000-25 & $\mathrm{B}$ & 1500 & 200 & 46.84 & 1.115 & 13.24 & $\mathrm{ST}$ \\
XG1000-25 & $\mathrm{C}$ & 300 & 200 & 46.84 & 0.2229 & 2.648 & $\mathrm{ST}$ \\
XG1000-25 & $\mathrm{D}$ & 150 & 200 & 46.84 & 0.1115 & 1.324 & $\mathrm{ST}$ \\
XG1000-25 & $\mathrm{E}^{*}$ & 30 & 200 & 46.84 & 0.02229 & 0.2648 & $\mathrm{ST}$ \\
P500-72 & $\mathrm{A}$ & 4000 & 226 & 99.23 & 5.822 & 15.41 & $\mathrm{E}$ \\
P500-72 & $\mathrm{B}$ & 2000 & 226 & 99.23 & 2.911 & 7.706 & $\mathrm{E}$ \\
P500-72 & $\mathrm{C}$ & 400 & 226 & 99.23 & 0.5822 & 1.541 & $\mathrm{E}$ \\
P500-72 & $\mathrm{D}^{*}$ & 200 & 226 & 99.23 & 0.2911 & 0.7706 & $\mathrm{E}$ \\
P500-72 & $\mathrm{E}^{*}$ & 90 & 253 & 99.23 & 0.1164 & 0.3082 & $\mathrm{E}$ \\
XG2000-50 & $\mathrm{A}$ & 4000 & 230 & 82.75 & 4.396 & 16.73 & $\mathrm{STE}$ \\
XG2000-50 & $\mathrm{B}$ & 2000 & 230 & 82.75 & 2.198 & 8.366 & $\mathrm{STE}$ \\
XG2000-50 & $\mathrm{C}$ & 400 & 230 & 82.75 & 0.4396 & 1.673 & $\mathrm{STE}$ \\
XG2000-50 & $\mathrm{D}^{*}$ & 200 & 230 & 82.75 & 0.2198 & 0.8366 & $\mathrm{STE}$ \\
\hline
\end{tabular}

Table 5: Experimental conditions: influence of ramp up acceleration rate. Experiments are conducted for samples expected to exhibit different behaviours: Newtonian (N) figure 6, Purely shear-thinning as described in figure 8 (ST) where elastic instabilities are suppressed by shear-thinning, purely elastic Boger fluid (E) (figure 7) with negligible shear-thinning, and shear-thinning dominated by elasticity (STE) figure $13 .{ }^{*}$ denotes experiments with $\Gamma_{0}<1$ also reported in table 2 .

In the Newtonian case (figure 19, left column, a), the flow maps are qualitatively similar for very different acceleration rates. However, the exact Reynolds number $\operatorname{Re}_{c}^{T V F}$ for the CF to TVF transition increases for the largest $\Gamma_{0}$ (a). Values of $\operatorname{Re}_{c}^{T F V}$ and $\lambda$ are plotted against $\mathrm{d} \Omega / \mathrm{d} t$ and $\Gamma_{0}$ in figure 20, for all $\mathrm{N}$ runs in table 5 (along with the critical Reynolds number for the onset of WTVF, $\operatorname{Re}_{c}^{W T V F}$, for the two lowest acceleration cases for which frequency maps can still be constructed). It appears that for $\Gamma_{0}<1$, the onset of TVF is not affected by the value of the acceleration rate as expected from the observations of Dutcher \& Muller (2009).

For the shear-thinning dominated case (figure 8), the flow maps are also not qualitatively affected. Note that in the fastest acceleration's case, one can clearly identify the waviness of Taylor vortices just from the flow map (d). Figure $20 \mathrm{ST}$ shows that for $\Gamma_{0}<3$ the critical Reynolds number for CF to TVF transition does not depend on $\Gamma_{0}$.

The results for the purely elastic case are qualitatively different in terms of flow states transition. From figure 19 (third column, c), it appears that the RSW is modified by the variations in acceleration rates. The Reynolds range in which it can be found reduces with increasing $\Gamma_{0}$, as illustrated in figure 20 where critical Reynolds numbers are plotted as a function of $\Gamma_{0}$. In the fastest acceleration case, it is difficult to tell whether the state observed from $\mathrm{Re}=100$ to about $\mathrm{Re}=120$ is TVF or RSW (this would require the use of frequency maps) but the flow signature is definitely different from low acceleration RSW. As in the $\mathrm{N}$ and ST cases, the onset of TVF is also delayed for high $\Gamma_{0}$ values, and $\operatorname{Re}_{c}^{T V F}$ 


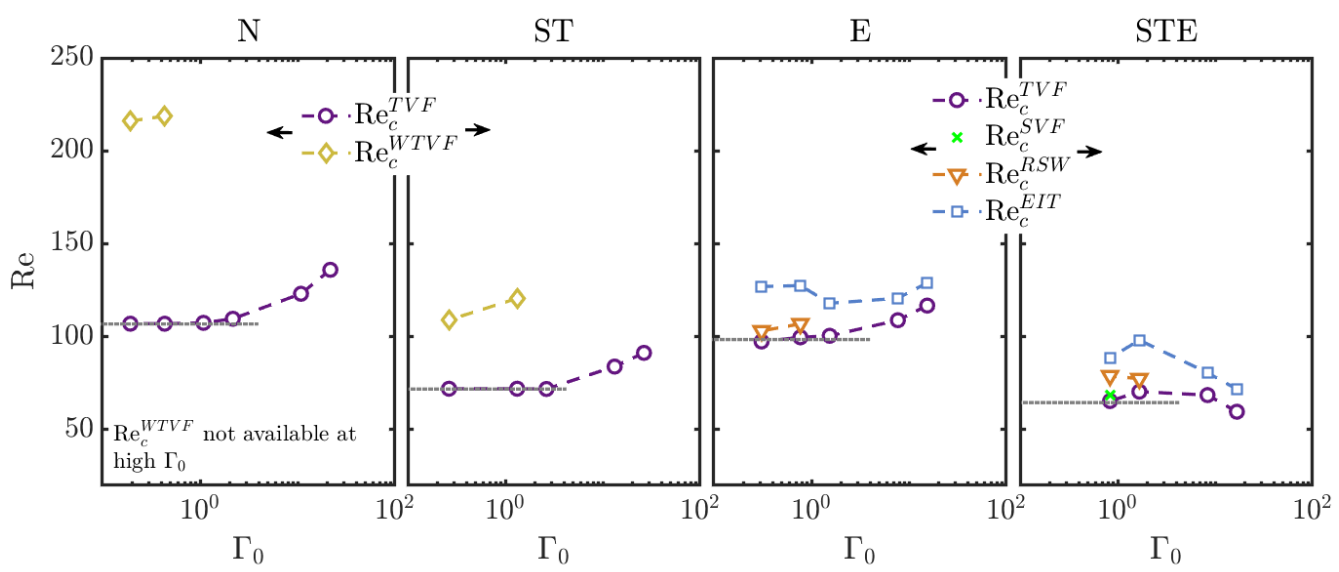

Figure 20: Values of critical Reynolds number for the onset of various flow states in the four investigated cases: Newtonian $(\mathrm{N})$, shear-thinning with Newtonian-like transitions (ST), purely elastic Boger (E) and shear-thinning with elastic flow patterns (STE).

can be assumed acceleration-independent for $\Gamma_{0}<1$. However values of $\operatorname{Re}_{c}^{E I T}$ are almost constant for all accelerations. This, in turn, leads to a narrowing of the Reynolds range left for the two intermediate regimes (TVF, RSW), i.e, regimes comprised between the base flow state (CF) and the ultimate elasto-inertial flow state (EIT). In other words, ramping up the inner cylinder at high acceleration rates leads to a "squeezing" of the intermediate transition steps in favour of an abrupt CF to EIT transition. Note that once again the "temporal" frequencies (patterns along Re axis) for RSW or EIT are more visible on the flow map in the fast cases, as the fast ramp up leads to capture only a limited number of waves period, but describe them with great resolution, even though that description spans a significant Re variation.

For the final case of a fluid where strong shear-thinning is dominated by elasticity (STE) we consider the XG2000-50 case studied previously (figures 13,14,15,16). Note that the XG1000-72 case has also been investigated and leads to results similar to the $\mathrm{E}$ case. As in the E case, suppression of intermediate regimes (TVF, RSW, SVF) is reported. Contrary to the previous cases however, the onset of the TVF flow is not affected as $\operatorname{Re}_{c}^{T V F}$ does not show any clear trend with $\Gamma_{0}$. On the other hand, it is the earlier onset of EIT that leads to the "squeezing" of the intermediate Reynolds range.

From a qualitative point of view and in terms of succession of flow states, the $\mathrm{N}$ and ST cases are acceleration-independent, but E and STE fluids may have intermediate regimes suppressed for $\Gamma_{0} \gg 1$. From a quantitative point of view, the CF to TVF transition is in general not affected by the acceleration rate for rates lower than unity (but higher acceleration rates may even be used in the ST case). To extend this acceleration study, it would be suitable to adapt the frequency maps analysis to highest acceleration rates, and adapt the dimensional acceleration rates to achieve constant non-dimensional acceleration rates throughout the ramp up for shear-thinning fluids, as done in Dutcher \& Muller (2013). 